\title{
Spectroscopic Studies and Thermal Analysis of New Azo Dyes Ligands and their Complexes with some Transition of Metal Ions
}

\author{
Abbas Ali Salih Al-Hamdani \\ Zainab Abd Al-Hadi Hasan \\ Department of Chemistry, College of Science for Women, University of Baghdad, \\ Iraq \\ E-mail: - Abbas_alhamadani@yahoo.co.uk
}

Received 20/4 /2015

Accepted 15/6 /2015

(i) $\Theta \Theta$

EY NG ND This work is licensed under a Creative Commons Attribution-NonCommercialNoDerivatives 4.0 International Licens

\begin{abstract}
:
New Azo ligands $\mathrm{HL}_{1}$ [2-Hydroxy-3-((5-mercapto-1,3,4-thiadiazol-2-yl)diazenyl)-1naphth aldehyde] and $\mathrm{HL}_{2}$ [3-((1,5-Dimethyl-3-oxo-2-phenyl-2,3-dihydro-1Hpyrazol-4-yl)diazenyl)-2-hydroxy-1-naphthaldehyde] have been synthesized from reaction (2-hydroxy-1-naphthaldehyde) and (5-amino-1,3,4-thiadiazole-2-thiol) for $\mathrm{HL}_{1}$ and (4-amino-1,5-dimethyl-2-phenyl-1H-pyrazol-3(2H)-one) for $\mathrm{HL}_{2}$. Then, its metal ions complexes are synthesized with the general formula; $\left[\mathrm{CrHL}_{1} \mathrm{Cl}_{3}\left(\mathrm{H}_{2} \mathrm{O}\right)\right.$ ], $\left[\mathrm{VOHL}_{1}\left(\mathrm{SO}_{4}\right)\right]\left[\mathrm{ML}_{1} \mathrm{Cl}\left(\mathrm{H}_{2} \mathrm{O}\right)\right]$ where $\mathrm{M}=\mathrm{Mn}(\mathrm{II}), \mathrm{Co}(\mathrm{II}), \mathrm{Ni}(\mathrm{II})$ and $\mathrm{Cu}(\mathrm{II})$, and general formula; $\left[\mathrm{Cr}\left(\mathrm{L}_{2}\right)_{2}\right] \mathrm{Cl}$ and $\left[\mathrm{M}\left(\mathrm{L}_{2}\right)_{2}\right]$ where $\mathrm{M}=\mathrm{VO}(\mathrm{II}), \mathrm{Mn}(\mathrm{II}), \mathrm{Co}(\mathrm{II}), \mathrm{Ni}(\mathrm{II})$ and $\mathrm{Cu}(\mathrm{II})$ are reported. The ligands and their metal complexes are characterized by phisco- chemical spectroscopic techniques (FT.IR, Mass, UV-Vis, ${ }^{1} \mathrm{H}$ and ${ }^{13} \mathrm{C}-\mathrm{NMR}$, TGA, (C.H.N.), molar conductivity, Atomic Absorbance, Chloride containing magnetic susceptibility). The spectral data suggest that the $\left(\mathrm{HL}_{1}\right)$ behaves as a bidentate ligand in all complexes, whereas $\left(\mathrm{HL}_{2}\right)$ behaves as a tridentate ligand for all complexes; bidentate ligand in Vanadium complex is coordinated with the metal ions through azo nitrogen and oxygen atoms. Theoretical studies of these ligands and their metal complexes in gas phase using Hyper chem.8. Studies of these compounds are prepared for their bacterial activity
\end{abstract}

Key words: Tri and Bidentate Ligands; Azo Ligand; Thermal Analysis and Kinetics Thermodynamic Parameters.

\section{Introduction:}

The coordination chemistry of transition al metals with ligands from the 5-amino1,3,4-thiadiazole-2-thiol, 2-hydroxy-1naphthaldehyde and 4-amino-1,5dimethyl-2-phenyl-1H-pyrazol-3(2H)one (aromatic heterocyclic components) has been of interest due to different bonding modes shown by these ligands with both electron rich and electron poor metals. Furthermore, they have been studied widely because of their excellent thermal and optical properties in applications such as optical recording medium [1-4], toner [5,6], ink-jet 
printing $[7,8]$, and oil-soluble lightfast dyes [9]. Several ligands containing 1,2,4-triazole are well known as drugs. For vorozole, letrozole, and anastrozole are non-steroidal drugs used for the treatment of cancer [10], while loreclezole is used as anticonvulsant [11] and fluconazole is used as an antimicrobial drug $[12,13]$. We report here, in the preparation and spectroscopic studies new ligands $\mathrm{HL}_{1}$ [2-Hydroxy-3-((5-mercapto-1,3,4thiadiazol-2-yl)diazenyl)-1-naphth aldehyde] and $\mathrm{HL}_{2}$ [3-((1,5-Dimethyl-3oxo-2-phenyl-2,3-dihydro-1 $H$-pyrazol4-yl)diazenyl)-2-hydroxy-1naphthaldehyde] and their complexes.

\section{Materials and Methods:}

All the following chemicals and reagents are of an analytical grade and used as supplied without any further purification (BDH) sigma Aldrich, and (Fluka).

IR spectra are obtained by using $\mathrm{KBr}$ discs (4000-400 $\mathrm{cm}^{-1}$ ) on Perkin -Elmer FT-IR spectrometer. The electronic spectra are carried out using a Cary 50 Conc. UV-Visible spectra are recorded using spectrophotometer. Thermal analysis studies of the compounds are performed on Perkin - Elmer pyris Diamond DTA / TG. Elemental microanalyses (C.H.N.S.) are performed by using a flash E A 1112 Series elemental analyzer. Chloride is determined by using potentiometer titration method on a 686-Titro processor - 665 Dosimat Metrohm Swiss. Conductivity measurements are made with DMSO solution by using a Jenway 4071 digital conductivity meter. The magnetic properties are measured by using (Jonson Mattey Catalytic system Division). Melting points are measured by using a Buchi SMP -20 capillary melting point apparatus, LCMass spectra by using (LC-Mass $100 \mathrm{P}$ Shimadzu).NMR spectra $\left({ }^{1} \mathrm{H}-,{ }^{13} \mathrm{C}-\right.$
NMR) by using Brucker AMX 400 $\mathrm{MHz}$ spectrometer. Atomic Absorption technique is used to determine the metal contents by using a Shimadzu (A. A) $680 \mathrm{G}$ atomic absorption spectrophotometer.

\section{Preparation of the Ligand 2-Hydroxy- 3-((5-mercapto-1,3,4-thiadiazol-2- yl)diazenyl)-1-Naphth Aldehyde $\left(\mathrm{HL}_{1}\right)$ :}

(1g, 9.17 mmole) of 5-amino-1,3,4thiadiazole-2-thiol is dissolved in a mixture of $(2 \mathrm{ml})$ hydrochloric acid, (10 $\mathrm{ml})$ ethanol and $(10 \mathrm{ml})$ distilled water, and diazotized at $5^{\circ} \mathrm{C}$ with sodium nitrite solution. The diazo solution 5amino-1,3,4-thiadiazole-2-diazonium is added dropwise with stirring to a cooled ethanolic solution of (1.5g, 9.17 mmole) of (2-hydroxy-1-naphthaldehyde $(25 \mathrm{ml})$ of $(1 \mathrm{M}) \mathrm{NaOH}$ solution is added to the orange colored mixture. The precipitate is filtered off and washed several times with (1:1) ethanol: water, mixture then left to dry. After that, the product is precipitated $(1.875 \mathrm{~g})$ yielding $(64.88 \%)$, m. p. $\left(145^{\circ} \mathrm{C}\right)$. The reaction is shown in Scheme (1).

IR $(\mathrm{KBr})$ for $\mathrm{HL}_{1}$ : $v($ azo $-\mathrm{N}=\mathrm{N}-)=$ 1520-1430, v $(\mathrm{O}-\mathrm{H})=3566, \mathrm{v}(\mathrm{C}-\mathrm{OH})=$ 1096, v(Ar-CH) = 3067, v(aldehyde C$\mathrm{H})=2889$, v(aldehyde $\mathrm{C}=\mathrm{O})=1645$, $v(\mathrm{~S}-\mathrm{H})=2597, \mathrm{v}(\mathrm{C}-\mathrm{S})=660-653$ and $v(C=N)=1635$ and $1575 \mathrm{~cm}^{-1}$. The GCMass for HL1 $(316 \mathrm{~m} / \mathrm{z})$ with $\left(\mathrm{C}_{13} \mathrm{H}_{8} \mathrm{~N}_{4} \mathrm{O}_{2} \mathrm{~S}_{2}\right), \quad(315 \mathrm{~m} / \mathrm{z}) \quad$ with $\left(\mathrm{C}_{13} \mathrm{H}_{7} \mathrm{~N}_{4} \mathrm{O}_{2} \mathrm{~S}_{2}\right), \quad(289 \mathrm{~m} / \mathrm{z}) \quad$ with $\left(\mathrm{C}_{11} \mathrm{H}_{5} \mathrm{~N}_{4} \mathrm{O}_{2} \mathrm{~S}_{2}\right), \quad(253 \mathrm{~m} / \mathrm{z}) \quad$ with $\left(\mathrm{C}_{11} \mathrm{HN}_{4} \mathrm{O}_{2} \mathrm{~S}\right), \quad(237 \mathrm{~m} / \mathrm{z}) \quad$ with $\left(\mathrm{C}_{11} \mathrm{HN}_{4} \mathrm{OS}\right), \quad(209 \mathrm{~m} / \mathrm{z}) \quad$ with $\left(\mathrm{C}_{11} \mathrm{HN}_{2} \mathrm{OS}\right), \quad(195 \mathrm{~m} / \mathrm{z}) \quad$ with $\left(\mathrm{C}_{11} \mathrm{HNOS}\right),(145 \mathrm{~m} / \mathrm{z})$ with $\left(\mathrm{C}_{11} \mathrm{NOS}\right)$, $(50 \mathrm{~m} / \mathrm{z})$ with $\left(\mathrm{C}_{7} \mathrm{NOS}\right)$ and $(39 \mathrm{~m} / \mathrm{z})$ with $\left(\mathrm{C}_{6} \mathrm{NOS}\right) .{ }^{1} \mathrm{H}-\mathrm{NMR}$ for $\mathrm{HL} 1$ (DMSO- $\left.d_{6}, \mathrm{ppm}\right): \delta 1.09\left(\mathrm{~s}, 6 \mathrm{H}, \mathrm{CCH}_{3}\right)$, $5.273(1 \mathrm{H}, \mathrm{s}, \mathrm{SH}), 3.929(1 \mathrm{H}, \mathrm{s}, \mathrm{OH})$, $9.123(1 \mathrm{H}, \mathrm{s}, \mathrm{O}=\mathrm{CH})$ aldehyde, 6.309$8.515(5 \mathrm{H}, \mathrm{m}$, naphthalene-H) arom. 
${ }^{13} \mathrm{C}-\mathrm{NMR}$ for HL1 (100.622 $\mathrm{MHz}$, DMSO- $\left.d_{6}\right)$ : shown in Figure 1- A.

Preparation of the Ligand 3-((1,5Dimethyl-3-oxo-2-phenyl-2,3-dihydro1H-pyrazol-4-yl) diazenyl)-2Hydroxy- 1-Naphthaldehyde $\left(\mathrm{HL}_{2}\right)$ : (1g, 4.923 mmole) of 4-amino-1,5dimethyl-2- phenyl- 1H-pyrazol-3 (2H)one is dissolved in a mixture of $(2 \mathrm{ml})$ hydrochloric acid, $(10 \mathrm{ml})$ ethanol and (10 $\mathrm{ml})$ distilled water, and diazotized at $5^{\circ} \mathrm{C}$ with sodium nitrite solution. The diazo solution (1,5-dimethyl-3-oxo-2phenyl-2,3-dihydro-1H-pyrazole-4diazonium is added drop wise with stirring to an ethanolic solution of (0.846 g, 4.923 mmole) of (2-hydroxy1-naphthaldehyde $(25 \mathrm{ml})$ of $(1 \mathrm{M})$ $\mathrm{NaOH}$ solution is added to the orange colored mixture. The precipitate is filtered off and washed several times with (1:1) ethanol: water, the mixture then is left to dry. After that, the product

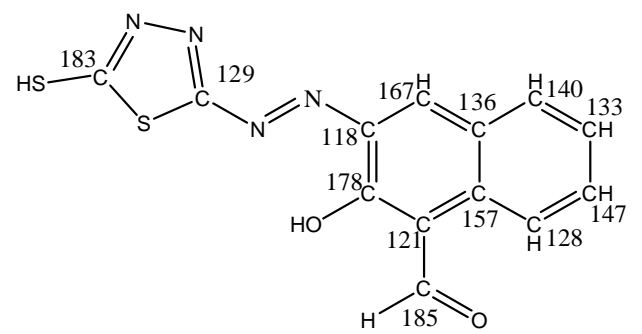

$\mathrm{HL}_{1}$

Figure (1): Structure of New Azo Dye Ligands
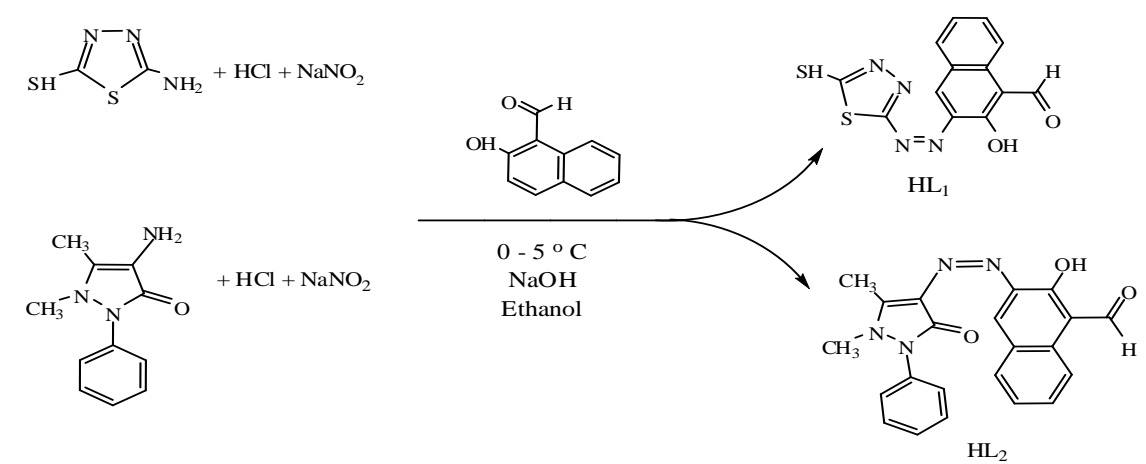

Scheme (1): Synthesis Route of New Azo Dye

Preparation of Metal Complexes (General Procedure) with Ligands:

An ethanolic solution of the ligand (1g, 3.164 mmole) HL1 and (2g, $5.18 \mathrm{mmol})$ is precipitated $(1.822 \mathrm{~g})$ yielding $(96 \%)$, $\mathrm{m}$. p. $\left(153-154^{\circ} \mathrm{C}\right)$. The reaction is shown in Scheme (1).

IR $(\mathrm{KBr})$ for $\mathrm{HL}_{2}$ : $\mathrm{v}($ azo $-\mathrm{N}=\mathrm{N}-)=1481$ and 1419, $\mathrm{v}(\mathrm{O}-\mathrm{H})=3429, \mathrm{v}(\mathrm{C}-\mathrm{OH})=$ $1087, v($ Ar $-\mathrm{CH})=3055$, v (aldehyde C$\mathrm{H})=2924, v($ aldehyde $\mathrm{C}=\mathrm{O})=1636$, $\mathrm{v}($ pyrazol ring $\mathrm{C}=\mathrm{O})=1643$ and $\mathrm{v}(\mathrm{C}=\mathrm{C})$ $=1593 \mathrm{~cm}^{-1}$. The GC-Mass for HL2 $(386 \mathrm{~m} / \mathrm{z})$ with $\left(\mathrm{C}_{22} \mathrm{H}_{18} \mathrm{~N}_{4} \mathrm{O}_{3}\right),(289 \mathrm{~m} / \mathrm{z})$ with $\left(\mathrm{C}_{14} \mathrm{H}_{17} \mathrm{~N}_{4} \mathrm{O}_{3}\right),(253 \mathrm{~m} / \mathrm{z})$ with $\left(\mathrm{C}_{14} \mathrm{H}_{13} \mathrm{~N}_{4} \mathrm{O}\right),(237 \mathrm{~m} / \mathrm{z})$ with $\left(\mathrm{C}_{14} \mathrm{H}_{13} \mathrm{~N}_{4}\right)$, $(209 \mathrm{~m} / \mathrm{z})$ with $\left(\mathrm{C}_{14} \mathrm{H}_{13} \mathrm{~N}_{2}\right),(195 \mathrm{~m} / \mathrm{z})$ with $\left(\mathrm{C}_{14} \mathrm{H}_{13} \mathrm{~N}\right),(145 \mathrm{~m} / \mathrm{z})$ with $\left(\mathrm{C}_{11} \mathrm{H}_{13}\right)$, $(96 \mathrm{~m} / \mathrm{z})$ with $\left(\mathrm{C}_{7} \mathrm{H}_{12}\right),(79 \mathrm{~m} / \mathrm{z})$ with $\left(\mathrm{C}_{6} \mathrm{H}_{7}\right)$ and $(39 \mathrm{~m} / \mathrm{z})$ with $\left(\mathrm{C}_{3} \mathrm{H}_{3}\right) .{ }^{1} \mathrm{H}$ NMR for HL2 (DMSO- $d_{6}$, ppm): $\delta 1.09$ $\left(\mathrm{s}, 6 \mathrm{H}, \mathrm{CCH}_{3}\right), 3.632(3 \mathrm{H}, \mathrm{s}, \mathrm{CH}), 1.407$ $(3 \mathrm{H}, \mathrm{s}, \mathrm{CH}), 4.329(1 \mathrm{H}, \mathrm{s}, \mathrm{OH})$ phenolic, $9.123(1 \mathrm{H}, \mathrm{s}, \mathrm{O}=\mathrm{CH})$ aldehyde, 7.059$7.815\left(10 \mathrm{H}, \mathrm{m}\right.$, aromatic-H). ${ }^{13} \mathrm{C}-\mathrm{NMR}$ for HL2 (100.622 MHz, DMSO- $\left.d_{6}\right)$ : show in Figure 1- B.

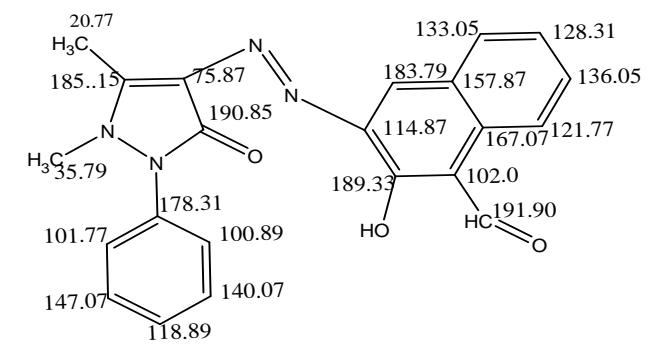

$\mathrm{HL}_{2}$ 
and $0.174 \mathrm{~g}(2.59 \mathrm{mmol})$ respectively, of $\mathrm{VOSO}_{4} \cdot \mathrm{H}_{2} \mathrm{O}, \mathrm{CrCl}_{3} \cdot 6 \mathrm{H}_{2} \mathrm{O}, \mathrm{MnCl}_{2} \cdot 4 \mathrm{H}_{2} \mathrm{O}$, $\mathrm{CoCl}_{2} \cdot 6 \mathrm{H}_{2} \mathrm{O}, \mathrm{NiCl}_{2} \cdot 6 \mathrm{H}_{2} \mathrm{O}$ and $\mathrm{CuCl}_{2}$ respectively dissolved in the buffer solution of the required PH. The solid is collected by filtration recrystallized from methanol dried at room temperature. Elemental micro analysis data color and yield for the compounds are given in Table (1).

The LC-Mass for $\mathrm{CrL}_{1}(493 \mathrm{~m} / \mathrm{z})$ with $\mathrm{M}=\left(\mathrm{C}_{13} \mathrm{H}_{10} \mathrm{~N}_{4} \mathrm{O}_{3} \mathrm{~S}_{2} \mathrm{CrCl}_{3}\right), \quad(360 \mathrm{~m} / \mathrm{z})$ with $\left(\mathrm{M}-\mathrm{C}_{2} \mathrm{H}_{3} \mathrm{Cl}_{3}\right)$ and $(119 \mathrm{~m} / \mathrm{z})$ with (M- $\mathrm{C}_{9} \mathrm{H}_{9} \mathrm{~N}_{2} \mathrm{O}_{2} \mathrm{~S}_{2}$ ). The LC-Mass for $\mathrm{VOL}_{1}(479 \mathrm{~m} / \mathrm{z})$ with $\mathrm{M}=$ $\left(\mathrm{C}_{13} \mathrm{H}_{8} \mathrm{~N}_{4} \mathrm{O}_{7} \mathrm{~S}_{3} \mathrm{~V}\right)$, (360m/z) with (M$\left.\mathrm{C}_{2} \mathrm{H}_{1} \mathrm{NSO}_{3}\right)$ and $(119 \mathrm{~m} / \mathrm{z})$ with (M$\mathrm{C}_{8} \mathrm{H}_{3} \mathrm{NS}_{2} \mathrm{O}_{4}$ ). The LC-Mass for $\mathrm{CoL}_{2}$ $(829.3 \mathrm{~m} / \mathrm{z})$ with $\mathrm{M}=\left(\mathrm{C}_{44} \mathrm{H}_{34} \mathrm{~N}_{8} \mathrm{O}_{6} \mathrm{Co}\right)$, $(430.3 \mathrm{~m} / \mathrm{z})$ with $\left(\mathrm{M}-\mathrm{C}_{21} \mathrm{H}_{11} \mathrm{~N}_{4} \mathrm{O}_{5}\right)$, $(429.3 \mathrm{~m} / \mathrm{z})$ with $(\mathrm{M}-\mathrm{H}),(205.2 \mathrm{~m} / \mathrm{z})$ with $\left(\mathrm{M}-\mathrm{C}_{18} \mathrm{H}_{8}\right),(204.2 \mathrm{~m} / \mathrm{z})$ with $(\mathrm{M}-\mathrm{H})$, $(79.4 \mathrm{~m} / \mathrm{z})$ with $\left(\mathrm{M}-\mathrm{CH}_{10} \mathrm{ON}_{2} \mathrm{Co}\right)$ and $(36 \mathrm{~m} / \mathrm{z})$ with $\left(\mathrm{M}-\mathrm{CH}_{3} \mathrm{~N}_{2}\right)$. The LC-Mass for $\mathrm{MnL}_{2} \quad(825.5 \mathrm{~m} / \mathrm{z})$ with $\mathrm{M}=\left(\mathrm{C}_{44} \mathrm{H}_{34} \mathrm{~N}_{8} \mathrm{O}_{6} \mathrm{Mn}\right),(430 \mathrm{~m} / \mathrm{z})$ with $(\mathrm{M}-$ $\left.\mathrm{C}_{22} \mathrm{H}_{11} \mathrm{~N}_{4} \mathrm{O}_{4}\right)$, (429m/z) with (M-H), $(205 \mathrm{~m} / \mathrm{z})$ with $\left(\mathrm{M}-\mathrm{C}_{12} \mathrm{H}_{8} \mathrm{~N}_{4} \mathrm{O}\right),(204 \mathrm{~m} / \mathrm{z})$ with $(\mathrm{M}-\mathrm{H}), \quad(79.4 \mathrm{~m} / \mathrm{z})$ with $(\mathrm{M}-$ $\left.\mathrm{C}_{4} \mathrm{H}_{6} \mathrm{OMn}\right)$ and $(36 \mathrm{~m} / \mathrm{z})$ with $\left(\mathrm{M}^{-} \mathrm{C}_{3} \mathrm{H}_{7}\right)$.
Elemental microanalysis and some physical properties of the $\left(\mathrm{HL}_{1}\right.$ and $\left.\mathrm{HL}_{2}\right)$ and their prepared complexes are shown in Table (1),

The composition of the complexes formed in solution has been established by the mole ratio method. In both cases, the result reveals $(1: 1)$ metal to ligand for $\mathrm{HL}_{1}$ but (1:2) metal to ligand for $\mathrm{HL}_{2}$ respectively (Figure - 2).

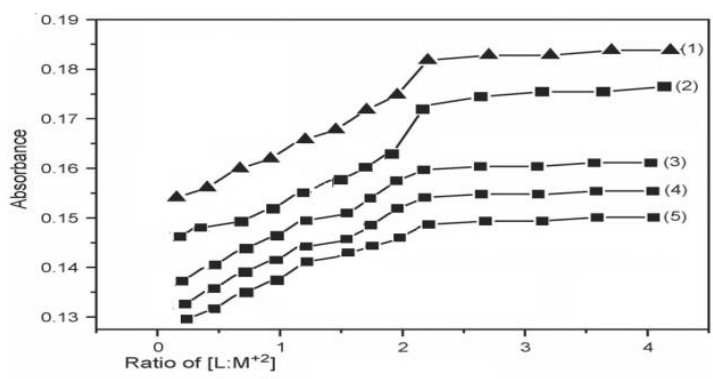

For $\mathrm{HL}_{1}$ complexes $\left(\mathrm{Co}^{2+}, \mathrm{Cu}^{2+}, \mathrm{Mn}^{2+}, \mathrm{Cr}^{3+}\right.$ and $\left.\mathrm{Ni}^{2+}\right)$

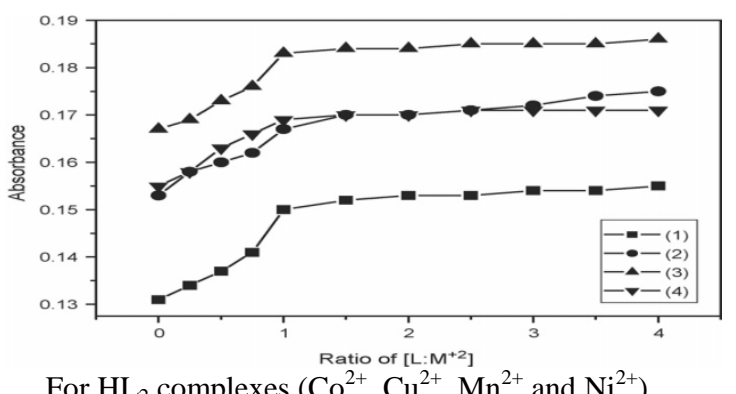

Fig. (2) Linear Relation between Molar Concentration and Absorbance

\section{Results and Discussion:}

Table (1) Analytical and Physical Data of the Ligands and their Complexes

\begin{tabular}{|c|c|c|c|c|c|c|c|c|c|}
\hline \multirow{2}{*}{ Compounds } & \multirow{2}{*}{$\begin{array}{l}\text { Formula } \\
\text { M. wt }\end{array}$} & \multirow{2}{*}{ Color } & \multirow{2}{*}{$\begin{array}{l}\text { M. P. } \\
{ }^{\circ} \mathrm{C}\end{array}$} & \multirow{2}{*}{$\begin{array}{c}\text { Yield } \\
\%\end{array}$} & \multicolumn{5}{|c|}{ Elemental analysis, Calc. (found) $\%$} \\
\hline & & & & & $\mathrm{C}$ & $\mathrm{H}$ & $\mathrm{N}$ & $\mathrm{M}$ & $\mathrm{Cl}$ \\
\hline $\mathrm{HL}_{1}$ & $\begin{array}{c}\mathrm{C}_{13} \mathrm{H}_{8} \mathrm{~N}_{4} \mathrm{O}_{2} \mathrm{~S}_{2} \\
316.36\end{array}$ & Orange & 145 & 64.879 & $\begin{array}{c}49.36 \\
(50.12) \\
\end{array}$ & $\begin{array}{c}2.55 \\
(3.07) \\
\end{array}$ & $\begin{array}{c}17.71 \\
(17.67) \\
\end{array}$ & - & - \\
\hline $\mathrm{HL}_{2}$ & $\begin{array}{c}\mathrm{C}_{22} \mathrm{H}_{18} \mathrm{~N}_{4} \mathrm{O}_{3} \\
386.40\end{array}$ & Orange & $153-154$ & 96 & $\begin{array}{c}68.38 \\
(67.88)\end{array}$ & $\begin{array}{c}4.70 \\
(5.02)\end{array}$ & $\begin{array}{c}14.50 \\
(14.54)\end{array}$ & - & - \\
\hline $\mathrm{VOL}_{1}$ & $\begin{array}{c}\mathrm{C}_{13} \mathrm{H}_{8} \mathrm{~N}_{4} \mathrm{O}_{7} \mathrm{~S}_{3} \mathrm{~V} \\
479.36\end{array}$ & Blue green & 220 & 75 & $\begin{array}{c}33.21 \\
(32.57)\end{array}$ & $\begin{array}{c}1.09 \\
(1.68)\end{array}$ & $\begin{array}{c}10.98 \\
(11.69)\end{array}$ & $\begin{array}{c}11.00 \\
(10.98)\end{array}$ & - \\
\hline $\mathrm{VO}\left(\mathrm{L}_{2}\right)_{2}$ & $\begin{array}{c}\mathrm{C}_{44} \mathrm{H}_{34} \mathrm{~N}_{8} \mathrm{O}_{7} \mathrm{~V} \\
837.73\end{array}$ & Green brown & $136-137$ & 79 & $\begin{array}{c}62.89 \\
(63.08)\end{array}$ & $\begin{array}{c}4.32 \\
(4.09)\end{array}$ & $\begin{array}{c}14.14 \\
(13.38)\end{array}$ & $\begin{array}{c}6.66 \\
(6.08)\end{array}$ & - \\
\hline $\mathrm{CrL}_{1}$ & $\begin{array}{c}\mathrm{C}_{13} \mathrm{H}_{10} \mathrm{~N}_{4} \mathrm{O}_{3} \mathrm{~S}_{2} \mathrm{CrCl}_{3} \\
492.73\end{array}$ & Green yellow & 188 & 80 & $\begin{array}{c}31.99 \\
(31.69)\end{array}$ & $\begin{array}{c}2.03 \\
(2.05)\end{array}$ & $\begin{array}{c}10.98 \\
(11.37)\end{array}$ & $\begin{array}{c}11.12 \\
(10.55)\end{array}$ & $\begin{array}{c}22.05 \\
(21.59)\end{array}$ \\
\hline $\mathrm{Cr}\left(\mathrm{L}_{2}\right)_{2}$ & $\begin{array}{c}\mathrm{C}_{44} \mathrm{H}_{34} \mathrm{~N}_{8} \mathrm{O}_{6} \mathrm{CrCl} \\
858.24\end{array}$ & Red brown & $127-130$ & 71 & $\begin{array}{c}61.58 \\
(62.01)\end{array}$ & $\begin{array}{c}3.99 \\
(4.12)\end{array}$ & $\begin{array}{c}13.06 \\
(13.86)\end{array}$ & $\begin{array}{c}6.06 \\
(6.34)\end{array}$ & $\begin{array}{c}4.13 \\
(4.10)\end{array}$ \\
\hline $\mathrm{MnL}_{1}$ & $\begin{array}{c}\mathrm{C}_{13} \mathrm{H}_{9} \mathrm{~N}_{4} \mathrm{O}_{3} \mathrm{~S}_{2} \mathrm{MnCl} \\
423.76\end{array}$ & Light yellow & $190 \mathrm{~d}$ & 70 & $\begin{array}{c}37.06 \\
(36.85)\end{array}$ & $\begin{array}{c}1.78 \\
(2.14)\end{array}$ & $\begin{array}{c}12.98 \\
(13.22)\end{array}$ & $\begin{array}{c}13.67 \\
(12.96)\end{array}$ & $\begin{array}{c}9.02 \\
(8.37)\end{array}$ \\
\hline $\operatorname{Mn}\left(\mathrm{L}_{2}\right)_{2}$ & $\begin{array}{c}\mathrm{C}_{44} \mathrm{H}_{34} \mathrm{~N}_{8} \mathrm{O}_{6} \mathrm{Mn} \\
825.73\end{array}$ & Deep yellow & $128-130$ & 76 & $\begin{array}{l}64.43 \\
(64.0) \\
\end{array}$ & $\begin{array}{c}7.05 \\
(6.65) \\
\end{array}$ & $\begin{array}{c}4.03 \\
(4.15) \\
\end{array}$ & $\begin{array}{c}14.04 \\
(13.57) \\
\end{array}$ & - \\
\hline $\mathrm{CoL}_{1}$ & $\begin{array}{c}\mathrm{C}_{13} \mathrm{H}_{9} \mathrm{~N}_{4} \mathrm{O}_{3} \mathrm{~S}_{2} \mathrm{CoCl} \\
427.75\end{array}$ & Light violet & $192 \mathrm{~d}$ & 74 & $\begin{array}{c}37.08 \\
(36.50)\end{array}$ & $\begin{array}{c}2.67 \\
(2.12) \\
\end{array}$ & $\begin{array}{c}12.78 \\
(13.10)\end{array}$ & $\begin{array}{c}13.21 \\
(13.78)\end{array}$ & $\begin{array}{c}7.87 \\
(8.29)\end{array}$ \\
\hline $\mathrm{Co}\left(\mathrm{L}_{2}\right)_{2}$ & $\begin{array}{c}\mathrm{C}_{44} \mathrm{H}_{34} \mathrm{~N}_{8} \mathrm{O}_{6} \mathrm{Co} \\
829.72\end{array}$ & Brown yellow & 144-146 & 73 & $\begin{array}{c}64.12 \\
(63.69)\end{array}$ & $\begin{array}{c}4.05 \\
(4.13)\end{array}$ & $\begin{array}{c}14.22 \\
(13.50)\end{array}$ & $\begin{array}{l}7.11 \\
7.10\end{array}$ & - \\
\hline $\mathrm{NiL}_{1}$ & $\begin{array}{c}\mathrm{C}_{13} \mathrm{H}_{9} \mathrm{~N}_{4} \mathrm{O}_{2} \mathrm{~S}_{2} \mathrm{NiCl} \\
427.51\end{array}$ & Light green & $199 \mathrm{~d}$ & 75 & $\begin{array}{c}35.98 \\
(36.52)\end{array}$ & $\begin{array}{c}3.04 \\
(2.12)\end{array}$ & $\begin{array}{c}12.87 \\
(13.11)\end{array}$ & $\begin{array}{c}13.98 \\
(13.73)\end{array}$ & $\begin{array}{c}8.98 \\
(8.29)\end{array}$ \\
\hline $\mathrm{Ni}\left(\mathrm{L}_{2}\right)_{2}$ & $\begin{array}{c}\mathrm{C}_{44} \mathrm{H}_{34} \mathrm{~N}_{8} \mathrm{O}_{6} \mathrm{Ni} \\
829.48\end{array}$ & Deep yellow & 201 & 78 & $\begin{array}{c}64.03 \\
(63.71)\end{array}$ & $\begin{array}{c}3.88 \\
(4.13)\end{array}$ & $\begin{array}{c}12.99 \\
(13.43)\end{array}$ & $\begin{array}{c}7.23 \\
(7.08)\end{array}$ & - \\
\hline $\mathrm{CuL}_{1}$ & $\begin{array}{c}\mathrm{C}_{13} \mathrm{H}_{9} \mathrm{~N}_{4} \mathrm{O}_{3} \mathrm{~S}_{2} \mathrm{CuCl} \\
432.36 \\
\end{array}$ & Yellow green & $229 \mathrm{~d}$ & 74 & $\begin{array}{c}37.02 \\
(36.11) \\
\end{array}$ & $\begin{array}{c}1.96 \\
(2.10) \\
\end{array}$ & $\begin{array}{c}13.45 \\
(12.96) \\
\end{array}$ & $\begin{array}{c}13.98 \\
(14.70) \\
\end{array}$ & $\begin{array}{c}8.76 \\
(8.20) \\
\end{array}$ \\
\hline $\mathrm{Cu}\left(\mathrm{L}_{2}\right)_{2}$ & $\begin{array}{c}\mathrm{C}_{44} \mathrm{H}_{34} \mathrm{~N}_{8} \mathrm{O}_{6} \mathrm{Cu} \\
834.34\end{array}$ & Brown & $220-222$ & 86 & $\begin{array}{c}63.64 \\
(63.34)\end{array}$ & $\begin{array}{c}4.43 \\
(4.11)\end{array}$ & $\begin{array}{c}12.99 \\
(13.43)\end{array}$ & $\begin{array}{c}7.24 \\
(7.62)\end{array}$ & 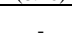 \\
\hline
\end{tabular}

$\mathrm{d}=$ decompose 
Infrared Spectral Studies of the Complexes

The main vibrational bands of the FTIR spectrum are assigned to the functional groups of the ligands $\mathrm{HL}_{1}$ and $\mathrm{HL}_{2}$. The spectrum of the $\mathrm{HL}_{1}$ shows strong bands at $1520-1430,3566$ and $1096 \mathrm{~cm}^{-1}$ that are assigned to the $v(\mathrm{~N}=\mathrm{N})$ and $v(\mathrm{O}-\mathrm{H})$ phenolic and $v(\mathrm{C}-\mathrm{OH})$ respectively [1415]. On complex formation, the bands of $v(\mathrm{~N}=\mathrm{N})$ are shifted to lower frequencies by (56-53 to 16-3); these shifts confirm the coordination of the $\mathrm{HL}_{1}$ through the oxygen of the phenol group and nitrogen of azo group with the metal ions. The spectrum of the $\mathrm{HL}_{2}$ shows strong bands at 1481-1419, 3429, 1087 and $1643 \mathrm{~cm}^{-1}$ that are assigned to the $v(\mathrm{~N}=\mathrm{N})$ and $v(\mathrm{OH})$ phenolic and $v(\mathrm{C}-\mathrm{OH})$ and $v(\mathrm{C}=\mathrm{O})$ pyrazol ringe respectively [1415]. On complexes formation, the bands of $v(\mathrm{~N}=\mathrm{N})$ and $v(\mathrm{C}=\mathrm{O})$ are shifted to lower frequencies by (12-4 to 39-8) and (11-6); these shifts confirm the coordination of the $\mathrm{HL}_{2}$ through the $(\mathrm{O})$ of the $(\mathrm{C}=\mathrm{O})$ group, $(\mathrm{N})$ of azo group and $(\mathrm{O})$ of $(\mathrm{O}-\mathrm{H})$ group with the metal ions but Vanadium ion coordination through the $(\mathrm{O})$ of the $(\mathrm{O}-\mathrm{H})$ group, and $(\mathrm{N})$ of azo group ion. Moreover, the spectra of the complexes exhibit weak bands between (505-586) $\mathrm{cm}^{-1}$ and (420$505) \mathrm{cm}^{-1}$ which are attributed to $v(\mathrm{M}-$ $\mathrm{N})$ and $v(\mathrm{M}-\mathrm{O})$ respectively [16-18]. The spectra of the Vanadium complexes exhibit weak band between (940-935) $\mathrm{cm}^{-1}$ which are attributed to $v(\mathrm{~V}=\mathrm{O})$. This indicates that the ligands are coordinated to the metal ions through the $\mathrm{N}$ and $\mathrm{O}$ atoms. Characteristic vibrations and assignments of the complexes are reported in Table (2).

Table (2) FTIR Spectral Data (Wave Number v $\mathrm{cm}^{-1}$ for the Complexes

\begin{tabular}{|c|c|c|c|c|c|c|c|c|c|c|}
\hline Compounds & $\begin{array}{c}\mathrm{H}_{2} \mathrm{O} \\
\text { Roking }\end{array}$ & $\mathrm{S}-\mathrm{H}$ & $\mathrm{C}-\mathrm{S}$ & $\begin{array}{c}\mathrm{OH} \\
\text { Phen. }\end{array}$ & $\begin{array}{l}\mathrm{C}=\mathrm{O} \\
\text { ring }\end{array}$ & $\begin{array}{c}\mathrm{C}=\mathrm{O} \\
\text { ald }\end{array}$ & $\begin{array}{c}\mathrm{C}=\mathrm{N} \\
\text { Triazol azo }\end{array}$ & $\mathrm{N}=\mathrm{N}$ & M-N & $\mathrm{M}-\mathrm{O}$ \\
\hline$\left[\mathrm{VO} \mathrm{HL} \mathrm{H}_{1}\left(\mathrm{SO}_{4}\right)\right]$ & - & 2590 & 654 & 3575 & - & 1644 & 1634 & 1465 & 534 & $\begin{array}{l}484 \\
423\end{array}$ \\
\hline$\left[\mathrm{VO}\left(\mathrm{L}_{2}\right)_{2}\right]$ & - & - & - & - & 1637 & 1633 & - & $\begin{array}{l}1473 \\
1456\end{array}$ & $\begin{array}{l}586 \\
536\end{array}$ & $\begin{array}{l}491 \\
474\end{array}$ \\
\hline$\left[\mathrm{Co} \mathrm{L}_{1} \mathrm{Cl}\left(\mathrm{H}_{2} \mathrm{O}\right)\right]$ & $\begin{array}{c}3450 \\
864\end{array}$ & 2570 & $\begin{array}{l}660 \\
653\end{array}$ & - & - & 1647 & 1635 & $\begin{array}{l}1465 \\
1400\end{array}$ & 530 & $\begin{array}{l}480 \\
424\end{array}$ \\
\hline$\left[\mathrm{Co}\left(\mathrm{L}_{2}\right)_{2}\right]$ & - & - & - & - & 1632 & 1629 & - & $\begin{array}{l}1477 \\
1457 \\
\end{array}$ & $\begin{array}{l}582 \\
536 \\
\end{array}$ & $\begin{array}{l}500 \\
474 \\
\end{array}$ \\
\hline$\left[\mathrm{Cu} \mathrm{L}{ }_{1} \mathrm{Cl}\left(\mathrm{H}_{2} \mathrm{O}\right)\right]$ & $\begin{array}{c}3451 \\
864 \\
\end{array}$ & 2585 & 653 & - & - & 1644 & 1634 & $\begin{array}{l}1465 \\
1404 \\
\end{array}$ & 534 & $\begin{array}{l}484 \\
423 \\
\end{array}$ \\
\hline$\left[\mathrm{Cu}\left(\mathrm{L}_{2}\right)_{2}\right]$ & - & - & - & - & 1635 & 1636 & - & $\begin{array}{l}1469 \\
1411 \\
\end{array}$ & $\begin{array}{l}580 \\
540 \\
\end{array}$ & $\begin{array}{l}490 \\
420 \\
\end{array}$ \\
\hline$\left[\mathrm{Ni} \mathrm{L}_{1} \mathrm{Cl}\left(\mathrm{H}_{2} \mathrm{O}\right)\right]$ & $\begin{array}{c}3448 \\
864 \\
\end{array}$ & 2573 & 654 & - & - & 1647 & 1637 & $\begin{array}{l}1467 \\
1403 \\
\end{array}$ & 533 & $\begin{array}{l}483 \\
423 \\
\end{array}$ \\
\hline$\left[\mathrm{Ni}\left(\mathrm{L}_{2}\right)_{2}\right]$ & - & - & - & - & 1637 & 1632 & - & $\begin{array}{l}1477 \\
1458\end{array}$ & $\begin{array}{l}582 \\
520\end{array}$ & $\begin{array}{l}505 \\
474\end{array}$ \\
\hline$\left[\mathrm{Cr} \mathrm{HL} \mathrm{HL}_{3}\left(\mathrm{H}_{2} \mathrm{O}\right)\right]$ & $\begin{array}{c}3415 \\
868\end{array}$ & 2583 & $\begin{array}{l}660 \\
653\end{array}$ & 3580 & - & 1644 & 1634 & $\begin{array}{l}1464 \\
1414\end{array}$ & 534 & $\begin{array}{l}484 \\
423\end{array}$ \\
\hline$\left[\mathrm{Cr}\left(\mathrm{L}_{2}\right)_{2}\right] \mathrm{Cl}$ & - & - & - & - & 1637 & 1633 & - & $\begin{array}{l}1475 \\
1455\end{array}$ & $\begin{array}{l}585 \\
505\end{array}$ & $\begin{array}{l}486 \\
475\end{array}$ \\
\hline$\left[\mathrm{Mn} \mathrm{L}_{1} \mathrm{Cl}\left(\mathrm{H}_{2} \mathrm{O}\right)\right]$ & $\begin{array}{c}3448 \\
864\end{array}$ & 2570 & $\begin{array}{l}664 \\
653\end{array}$ & - & - & 1644 & 1634 & $\begin{array}{l}1465 \\
1414\end{array}$ & 534 & $\begin{array}{l}484 \\
423\end{array}$ \\
\hline$\left[\mathrm{Mn}\left(\mathrm{L}_{2}\right)_{2}\right]$ & - & - & - & - & 1638 & 1638 & 1632 & $\begin{array}{l}1477 \\
1458\end{array}$ & $\begin{array}{l}582 \\
520\end{array}$ & $\begin{array}{l}488 \\
474\end{array}$ \\
\hline
\end{tabular}

Electronic Spectral, Magnetic Moments, and Conductivity Measurements

The electronic spectra of the ligands $\mathrm{HL}_{1}$ and $\mathrm{HL}_{2}$ exhibits intense absorption at $(265)$ and $(315,357,420) \mathrm{nm}$ for $\mathrm{HL}_{1}$ and $(267),(324,346,388) \mathrm{nm}$ for $\mathrm{HL}_{2}$ attributed to $\pi \rightarrow \pi^{*}$ and $\mathrm{n} \rightarrow \pi^{*}$ transitions respectively $[18,19]$.
The electronic spectra of the Co(II) complexes with $\mathrm{HL}_{1}$ and $\mathrm{HL}_{2}$ ligands exhibit one and three absorption band at $677 \mathrm{~nm}$ and $(615,678,909) \mathrm{nm}$ which are attributed to ${ }^{4} \mathrm{~A}_{2} \rightarrow{ }^{4} \mathrm{~T}_{1 \mathrm{P}}$ and ${ }^{4} \mathrm{~T}_{1 \mathrm{~g}} \rightarrow{ }^{4} \mathrm{~T}_{1 \mathrm{~g}(\mathrm{P})}$, ${ }^{4} \mathrm{~T}_{1 \mathrm{~g}} \rightarrow{ }^{4} \mathrm{~A}_{2 \mathrm{~g}(\mathrm{~F})} \quad$ and $\quad{ }^{4} \mathrm{~T}_{1 \mathrm{~g}} \rightarrow{ }^{4} \mathrm{~A}_{2 \mathrm{gF}}$ respectively. Furthermore, the magnetic moment of the $\operatorname{Co}(\mathrm{II})(d 7)$ complexes 
was found to be 4.1 and 4.8 B.M., All the above mentioned data correspond to an tetrahedral and octahedral geometry respectively [18-20]. The electronic spectra of the $\mathrm{Ni}$ (II) complexes with $\mathrm{HL}_{1}$ and $\mathrm{HL}_{2}$ ligands exhibit one and three absorption band at $797 \mathrm{~nm}$ and $(597,690,780) \mathrm{nm}$ which are attributed to ${ }^{3} \mathrm{~T}_{1} \rightarrow{ }^{3} \mathrm{~T}_{1 \mathrm{P}}$ and ${ }^{3} \mathrm{~A}_{2 \mathrm{~g}} \rightarrow{ }^{3} \mathrm{~T}_{1 \mathrm{gP}},{ }^{3} \mathrm{~A}_{1 \mathrm{~g}} \rightarrow{ }^{3} \mathrm{~T}_{1 \mathrm{gF}}$ and ${ }^{3} \mathrm{~A}_{1 \mathrm{~g}} \rightarrow{ }^{3} \mathrm{~T}_{2 \mathrm{gF}}$ respectively.Furthermore , the magnetic moment of the $\mathrm{Ni}(\mathrm{II})(\mathrm{d} 8)$ complexes is found to be 3.9 and 2.80 B.M., All the above mentioned data correspond to an tetrahedral and octahedral geometry respectively [1820]. The electronic spectra of the $\mathrm{Cu}$ (II) complexes exhibit one and two absorption band with $\mathrm{HL}_{1}$ and $\mathrm{HL}_{2}$ ligands at $953 \mathrm{~nm}$ and (450 and 540) nm which attributed to ${ }^{2} \mathrm{~T}_{2} \rightarrow{ }^{2} \mathrm{E}$ and ${ }^{2} \mathrm{~B}_{1 \mathrm{~g}} \rightarrow$ ${ }^{2} \mathrm{~A}_{1 \mathrm{~g}}$ and ${ }^{2} \mathrm{~B}_{1 \mathrm{~g}} \rightarrow{ }^{2} \mathrm{~B}_{2 \mathrm{~g}}$ respectively. Furthermore, the magnetic moment of the $\mathrm{Cu}(\mathrm{II})(d 9)$ complexes is found to be 2.01 and 1.9 B.M., All the above mentioned data correspond to an tetrahedral and octahedral geometry respectively [18]. The electronic spectra of the $\mathrm{Cr}$ (III) complexes exhibit three absorption band with $\mathrm{HL}_{1}$ and $\mathrm{HL}_{2}$ ligands at $(535,645,905) \mathrm{nm}$ and $(601,736,864) \mathrm{nm}$ which attributed to ${ }^{4} \mathrm{~A}_{2 \mathrm{~g}} \rightarrow{ }^{3} \mathrm{~T}_{1 \mathrm{gP}},{ }^{4} \mathrm{~A}_{2 \mathrm{~g}} \rightarrow{ }^{3} \mathrm{~T}_{1 \mathrm{gF}}$ and ${ }^{4} \mathrm{~A}_{2 \mathrm{~g}} \rightarrow{ }^{3} \mathrm{~T}_{2 \mathrm{gF}}$ respectively [20-22]. Furthermore, the magnetic moment of the $\mathrm{Cr}(\mathrm{III})(d 3)$ complexes is found to be 3.87 and 3.77 B.M., All the above mentioned data correspond to an octahedral geometry respectively [22]. The electronic spectra of the VO(II) complexes exhibit two absorption band with $\mathrm{HL}_{1}$ and $\mathrm{HL}_{2}$ ligands at (515 and 618) $\mathrm{nm}$ and (815 and 915) $\mathrm{nm}$ which are attributed to ${ }^{2} \mathrm{~B}_{2 \mathrm{~g}} \rightarrow{ }^{2} \mathrm{~B}_{1 \mathrm{~g}}$ and ${ }^{2} \mathrm{~B}_{2 \mathrm{~g}} \rightarrow{ }^{2} \mathrm{E}_{\mathrm{g}}$ respectively [20-22]. Furthermore, the magnetic moment of the $\mathrm{VO}$ (II) (dl) complexes is found to be 1.81 and 1.86 B.M., All the above mentioned data correspond to an square pyramidal geometry respectively [22]. The electronic spectra of the $\mathrm{Mn}(\mathrm{II})$ complexes exhibits two and three absorption band with $\mathrm{HL}_{1}$ and $\mathrm{HL}_{2}$ ligands at (696 and 850) $\mathrm{nm}$ and (540, $650,905) \mathrm{nm}$ which are attributed to $\left({ }^{6} \mathrm{~A}_{1} \rightarrow{ }^{4} \mathrm{~A}_{1}+{ }^{4} \mathrm{E}_{\mathrm{G}},{ }^{6} \mathrm{~A}_{1} \rightarrow{ }^{4} \mathrm{~T}_{1 \mathrm{G}}\right) \quad$ and $\left({ }^{6} \mathrm{~A}_{1} \rightarrow{ }^{4} \mathrm{~T}_{2} \mathrm{~g}, \quad{ }^{6} \mathrm{~A}_{1} \rightarrow{ }^{4} \mathrm{~T}_{1} \mathrm{~g}, \quad{ }^{6} \mathrm{~A}_{1} \rightarrow{ }^{4} \mathrm{E}_{1} \mathrm{~g}\right)$ respectively. Furthermore, the magnetic moment of the $\mathrm{Mn}(\mathrm{II})(d 5)$ complexes is found to be 5.45 and 5.01 B.M., All the above mentioned data correspond to an tetrahedral and octahedral geometry respectively [18].

The molar conductivity value of the complexes is consistent with nonelectrolytes for all complexes and 1:1 electrolytes $\mathrm{Cr}(\mathrm{III})$ complex with $\mathrm{HL}_{2}$. See structure for the complexes Figure (3).

Table (3) Electronic Data, $\mu_{\mathrm{eff}}$ and Molar Conductivity for the Complexes

\begin{tabular}{|c|c|c|c|c|c|c|c|}
\hline Complexes & $\Lambda \mathrm{nm}$ & $\bar{v} \mathrm{~cm}^{-1}$ & $\varepsilon \mathrm{L} \cdot \mathrm{mol}^{-1} \mathrm{~cm}^{-1}$ & Assignment & $\Omega \mathrm{Ohm}^{-1} \mathrm{~cm}^{2} \mathrm{~mol}^{-1}$ & $\mu_{\text {eff }}$ B.M & Suggested Formula \\
\hline$\left[\mathrm{VO} \mathrm{HL}_{1}\left(\mathrm{SO}_{4}\right)\right]$ & $\begin{array}{l}515 \\
618\end{array}$ & $\begin{array}{l}19417.4 \\
16181.2\end{array}$ & $\begin{array}{l}206 \\
121\end{array}$ & $\begin{array}{l}{ }^{2} \mathrm{~B}_{2 g} \rightarrow{ }^{2} B_{1 g} \\
{ }^{2} B_{2 g} \rightarrow{ }^{2} E_{g}\end{array}$ & 15.7 & 1.81 & $\begin{array}{c}\text { Square } \\
\text { Pyramidal }\end{array}$ \\
\hline$\left[\mathrm{Cr} \mathrm{HL}_{1} \mathrm{Cl}_{3}\left(\mathrm{H}_{2} \mathrm{O}\right)\right]$ & $\begin{array}{l}535 \\
645 \\
905\end{array}$ & $\begin{array}{l}18691.5 \\
15503.8 \\
11049.7\end{array}$ & $\begin{array}{c}64 \\
56 \\
8\end{array}$ & $\begin{array}{c}{ }^{4} \mathrm{~A}_{2 \mathrm{~g}} \rightarrow{ }^{3} \mathrm{~T}_{1 \mathrm{~g}(\mathrm{P})} \\
{ }^{4} \mathrm{~A}_{2 \mathrm{~g}} \rightarrow{ }^{3} \mathrm{~T}_{1 \mathrm{~g}(\mathrm{~F})} \\
{ }^{4} \mathrm{~A}_{2 \mathrm{~g}} \rightarrow{ }^{3} \mathrm{~T}_{2 \mathrm{~g}}\end{array}$ & 18 & 3.87 & Octahedral \\
\hline$\left[\mathrm{Mn} \mathrm{L}_{1} \mathrm{Cl}\left(\mathrm{H}_{2} \mathrm{O}\right)\right]$ & $\begin{array}{l}696 \\
850 \\
\end{array}$ & $\begin{array}{l}14367.8 \\
11764.7 \\
\end{array}$ & $\begin{array}{c}216 \\
68 \\
\end{array}$ & $\begin{array}{c}{ }^{6} \mathrm{~A}_{1} \rightarrow{ }^{4} \mathrm{~A}_{1}+{ }^{4} \mathrm{E}_{(\mathrm{G})} \\
{ }^{6} \mathrm{~A}_{1} \rightarrow{ }^{4} \mathrm{~T}_{1 \mathrm{G}}\end{array}$ & 11.6 & 5.45 & Tetrahedral \\
\hline$\left[\mathrm{Co} \mathrm{L} \mathrm{L}_{1} \mathrm{Cl}\left(\mathrm{H}_{2} \mathrm{O}\right)\right]$ & 677 & 14771.0 & 306 & ${ }^{4} \mathrm{~A}_{2} \rightarrow{ }^{4} \mathrm{~T}_{1(\mathrm{P})}$ & 13.76 & 4.10 & Tetrahedral \\
\hline$\left[\mathrm{Ni} \mathrm{L} \mathrm{L}_{1} \mathrm{Cl}\left(\mathrm{H}_{2} \mathrm{O}\right)\right]$ & 797 & 12547.0 & 102 & ${ }^{3} \mathrm{~T}_{1} \rightarrow{ }^{3} \mathrm{~T}_{1(\mathrm{P})}$ & 9.21 & 3.9 & Tetrahedral \\
\hline$\left[\mathrm{Cu} \mathrm{L} \mathrm{L}_{1} \mathrm{Cl}\left(\mathrm{H}_{2} \mathrm{O}\right)\right]$ & 953 & 10493.1 & 528 & ${ }^{2} \mathrm{~T}_{2} \rightarrow{ }^{2} \mathrm{E}$ & 14.8 & 1.63 & Tetrahedral \\
\hline$\left[\mathrm{VO}\left(\mathrm{L}_{2}\right)_{2}\right]$ & $\begin{array}{l}815 \\
915 \\
\end{array}$ & $\begin{array}{l}12269.9 \\
10928.9\end{array}$ & $\begin{array}{c}102 \\
17 \\
\end{array}$ & $\begin{array}{l}{ }^{2} \mathrm{~B}_{2 \mathrm{~g}} \rightarrow{ }^{2} \mathrm{~B}_{1 \mathrm{~g}} \\
{ }^{2} \mathrm{~B}_{2 \mathrm{~g}} \rightarrow{ }^{2} \mathrm{E}_{\mathrm{g}}\end{array}$ & 11.42 & 1.86 & $\begin{array}{c}\text { Square } \\
\text { Pyramidal }\end{array}$ \\
\hline$\left[\mathrm{Cr}\left(\mathrm{L}_{2}\right)_{2}\right] \mathrm{Cl}$ & $\begin{array}{l}601 \\
736 \\
864 \\
\end{array}$ & $\begin{array}{l}16638.9 \\
13586.9 \\
11574.0 \\
\end{array}$ & $\begin{array}{l}406 \\
285 \\
154 \\
\end{array}$ & $\begin{array}{l}{ }^{4} \mathrm{~A}_{2 g} \rightarrow{ }^{3} \mathrm{~T}_{1 g}(\mathrm{P}) \\
{ }^{4} \mathrm{~A}_{2 \mathrm{~g}} \rightarrow{ }^{3} \mathrm{~T}_{1 \mathrm{~g}} \\
{ }^{4} \mathrm{~A}_{2 \mathrm{~g}} \rightarrow{ }^{3} \mathrm{~T}_{2 \mathrm{~g}}\end{array}$ & 36.7 & 3.77 & Octahedral \\
\hline$\left[\mathrm{Mn}\left(\mathrm{L}_{2}\right)_{2}\right]$ & $\begin{array}{l}540 \\
650 \\
905\end{array}$ & $\begin{array}{l}18518.5 \\
15384.6 \\
11049.7\end{array}$ & $\begin{array}{c}125 \\
102 \\
80\end{array}$ & $\begin{array}{l}{ }^{6} \mathrm{~A}_{1} \rightarrow{ }^{4} \mathrm{~T}_{2 \mathrm{~g}} \\
{ }^{6} \mathrm{~A}_{1} \rightarrow{ }^{4} \mathrm{~T}_{1 \mathrm{~g}} \\
{ }^{6} \mathrm{~A}_{1} \rightarrow{ }^{4} \mathrm{E}_{\mathrm{g}}\end{array}$ & 15.08 & 5.01 & Octahedral \\
\hline$\left[\mathrm{Co}\left(\mathrm{L}_{2}\right)_{2}\right]$ & $\begin{array}{l}615 \\
678 \\
909 \\
\end{array}$ & $\begin{array}{l}16260.1 \\
14749.2 \\
11001.1\end{array}$ & $\begin{array}{c}61 \\
106 \\
9\end{array}$ & $\begin{array}{l}{ }^{4} \mathrm{~T}_{1 \mathrm{~g}} \rightarrow{ }^{4} \mathrm{~T}_{1 \mathrm{~g}}(\mathrm{P}) \\
{ }^{4} \mathrm{~T}_{1 \mathrm{~g}} \rightarrow{ }^{4} \mathrm{~A}_{2 \mathrm{~g}(\mathrm{~F})} \\
\left.{ }^{4} \mathrm{~T}_{1 \mathrm{~g}} \rightarrow{ }^{4} \mathrm{~T}_{2 \mathrm{~g}} \mathrm{~F}\right)\end{array}$ & 7.78 & 4.39 & Octahedral \\
\hline$\left[\mathrm{Ni}\left(\mathrm{L}_{2}\right)_{2}\right]$ & $\begin{array}{l}597 \\
690 \\
780 \\
\end{array}$ & $\begin{array}{l}16750.4 \\
14492.7 \\
12820.5 \\
\end{array}$ & $\begin{array}{c}86 \\
146 \\
79 \\
\end{array}$ & $\begin{aligned}{ }^{3} \mathrm{~A}_{2 \mathrm{~g}} & \rightarrow{ }^{3} \mathrm{~T}_{1 \mathrm{~g}(\mathrm{P})} \\
{ }^{3} \mathrm{~A}_{1 \mathrm{gg}} & \rightarrow{ }^{3} \mathrm{~T}_{1 \mathrm{~g}} \\
{ }^{3} \mathrm{~A}_{1 \mathrm{gg}} & \rightarrow{ }^{3} \mathrm{~T}_{2 \mathrm{~g}}\end{aligned}$ & 6.9 & 3.24 & Octahedral \\
\hline$\left[\mathrm{Cu}\left(\mathrm{L}_{2}\right)_{2}\right]$ & $\begin{array}{l}450 \\
540 \\
909\end{array}$ & $\begin{array}{l}22222.2 \\
18518.5 \\
11001.1\end{array}$ & $\begin{array}{c}630 \\
208 \\
50\end{array}$ & $\begin{array}{l}{ }^{2} \mathrm{~B}_{1} \mathrm{~g} \rightarrow{ }^{2} \mathrm{~A}_{1} \mathrm{~g} \\
{ }^{2} \mathrm{~B}_{1} \mathrm{~g} \rightarrow{ }^{2} \mathrm{~B}_{2} \mathrm{~g} \\
{ }^{2} \mathrm{~B}_{1} \mathrm{~g} \rightarrow{ }^{2} \mathrm{Eg}\end{array}$ & 8.08 & 1.9 & Octahedral \\
\hline
\end{tabular}




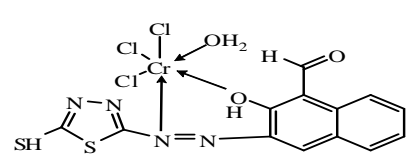

1
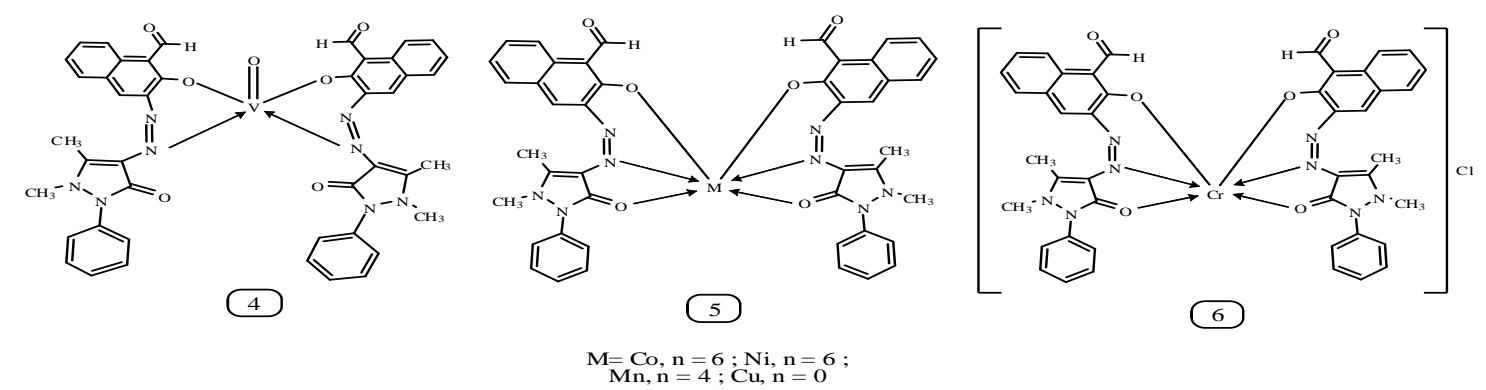

Fig. (3) Structure of the Complexes

Thermal Analyses TGA and Kinetic Studies:-

The results of thermo gravimetric analyses of ligands and complexes are given in Table (4). The thermograms have been carried out in the range of ${ }^{25}-700{ }^{\circ} \mathrm{C}$ at a heating rate of 10 ${ }^{\circ} \mathrm{C} / \mathrm{min}$ in nitrogen atmosphere. They show an agreement in weight loss between their results obtained from the thermal decomposition and the calculated values, which supports the results of elemental analysis and confirms the suggested formulae. Thus, the ligands and complexes show a common general behavior as show in Scheme (1). Coats-Redfern is the method mentioned in the literature related to decomposition kinetics studies; this method is applied in this study [23,24]. Kinetic parameters are calculated by employing the CoatsRedfern equations which are summarized in Table (5). The CoatsRedfern equation may be written as the following:

$$
\begin{aligned}
& \log \left[\frac{1-(1-\alpha)^{1-n}}{T^{2}(1-n)}\right]= \log \frac{Z R}{q E}\left[1-\frac{2 R T}{E}\right] \\
&-\frac{E}{2.303 R T} n \neq 1 \text { for complex } \\
& \log \left[\frac{-\log (1-\alpha)}{T^{2}}\right]= \log \left[\frac{A R}{\beta E}\left(1-\frac{2 R T}{E}\right] \frac{E}{2.303 R T}\right. \\
& n \neq 1 \text { for ligands }
\end{aligned}
$$

The activation entropy $\Delta S^{*}$, the activation enthalpy $\Delta H^{*}$ and the free energy of activation $\Delta \mathrm{G}^{*}$ were calculated using the following equations:

$$
S^{*}=2: 303(\log A h / K T) R ; H^{*}=E^{*}-R T ; G^{*}
$$$$
=H^{*}-T_{S} S^{*} \cdots 3
$$

Where $\mathrm{K}$ and $\mathrm{h}$ are the Boltzmann's and Planck's constants, respectively. The calculated values of $\Delta \mathrm{E}^{*}, \Delta \mathrm{S}^{*}, \Delta \mathrm{H}^{*}$ and $\Delta \mathrm{G}^{*}$ for the dehydration and the decomposition steps are given in table (5). 
Table (4) Thermal Analysis Data of the Free Ligands and Metal Complexes

\begin{tabular}{|c|c|c|c|c|c|c|c|}
\hline \multirow[t]{3}{*}{ Compounds } & \multicolumn{5}{|c|}{ TGA } & \multicolumn{2}{|c|}{ DTA } \\
\hline & \multirow{2}{*}{ TG range $\left({ }^{\circ} \mathrm{C}\right)$} & \multirow{2}{*}{$\begin{array}{c}\text { DTG } \\
\max \left({ }^{\circ} \mathrm{C}\right)\end{array}$} & \multicolumn{2}{|c|}{$\%$ Estimated (calculated) } & \multirow{2}{*}{ Assignment } & \multirow{2}{*}{ Tdta } & \multirow{2}{*}{ Peak } \\
\hline & & & Mass loss & Total mass lose & & & \\
\hline $\mathrm{HL}_{1}$ & $\begin{array}{l}164.2-235.5 \\
235.5-599.5\end{array}$ & $\begin{array}{l}210.1 \\
566.5\end{array}$ & $\begin{array}{l}28.88(28.47) \\
68.72(67.33)\end{array}$ & $\begin{array}{c}97.6 \\
(96.2)\end{array}$ & $\begin{array}{l}\mathrm{CO}_{2}+\mathrm{H}_{4} \mathrm{~N}_{3} \\
\mathrm{C}_{11} \mathrm{H}_{4} \mathrm{NS}_{2}\end{array}$ & $\begin{array}{c}185 \\
503.4\end{array}$ & $\begin{array}{l}\text { endo } \\
\text { endo }\end{array}$ \\
\hline $\mathrm{HL}_{2}$ & $\begin{array}{l}199.1-313.7 \\
313.7-589.3\end{array}$ & $\begin{array}{l}287.9 \\
524.4\end{array}$ & $\begin{array}{c}29.8(29.53) \\
69.54(70.47)\end{array}$ & $\begin{array}{l}99.34 \\
(100)\end{array}$ & $\begin{array}{l}\mathrm{C}_{4} \mathrm{H}_{6} \mathrm{~N}_{2} \mathrm{O}_{2} \\
\mathrm{C}_{18} \mathrm{H}_{12} \mathrm{~N}_{2} \mathrm{O}\end{array}$ & $\begin{array}{l}267.7 \\
464.7\end{array}$ & $\begin{array}{l}\text { exo } \\
\text { exo }\end{array}$ \\
\hline $\mathrm{MnL}_{1}$ & $\begin{array}{c}51.6-109.3 \\
178.8-257.9 \\
297-408.9 \\
408.9-519.7 \\
543.8-699.6\end{array}$ & $\begin{array}{l}95.13 \\
241.5 \\
358.8 \\
467.8 \\
614.3\end{array}$ & $\begin{array}{c}4.247(4.141) \\
8.74(9.082) \\
12.15(11.367) \\
18.14(18.19) \\
54.462(54.764)\end{array}$ & $\begin{array}{c}97.74 \\
(97.544)\end{array}$ & $\begin{array}{c}-\mathrm{H}_{2} \mathrm{O} \\
-\mathrm{Cl} \\
-\mathrm{CSH} \\
-\mathrm{CHNS} \\
-\mathrm{C}_{7} \mathrm{H}_{3} \mathrm{~N}_{3} \mathrm{O} \\
\mathrm{C}_{4} \mathrm{H}_{2} \mathrm{OMn}\end{array}$ & $\begin{array}{c}105 \\
251 \\
360.7 \\
484.1 \\
635.9\end{array}$ & $\begin{array}{l}\text { end } \\
\text { end } \\
\text { end } \\
\text { end } \\
\text { end }\end{array}$ \\
\hline $\mathrm{CuL}_{1}$ & $\begin{array}{c}33.5-132.8 \\
133-433.7 \\
462.9-699.4\end{array}$ & $\begin{array}{c}70.32 \\
319 \\
611.6\end{array}$ & $\begin{array}{c}4.163(5.271) \\
22.794(22.821) \\
70.02(70.096)\end{array}$ & $\begin{array}{c}96.977 \\
(98.188)\end{array}$ & $\begin{array}{c}-\mathrm{H}_{2} \mathrm{O} \\
-\mathrm{CHNSCl} \\
-\mathrm{C}_{12} \mathrm{H}_{6} \mathrm{~N}_{3} \mathrm{~S} \\
\mathrm{CuO}\end{array}$ & $\begin{array}{c}69.44 \\
314.3 \\
605.66\end{array}$ & $\begin{array}{l}\text { endo } \\
\text { endo } \\
\text { endo }\end{array}$ \\
\hline $\mathrm{CrL}_{1}$ & $\begin{array}{c}33.6-147.6 \\
305.4-399.7 \\
430.1-699.3\end{array}$ & $\begin{array}{l}72.03 \\
359 \\
476\end{array}$ & $\begin{array}{c}7.25(7.647) \\
32.303(31.601) \\
53.009(52.31)\end{array}$ & $\begin{array}{c}92.562 \\
(91.558)\end{array}$ & $\begin{array}{c}-\mathrm{H}_{2} \mathrm{O}+1 / 2 \mathrm{Cl} \\
-\mathrm{CHNSCl}_{2.5} \\
-\mathrm{C}_{11} \mathrm{H}_{4} \mathrm{~N}_{2} \\
\mathrm{CH}_{3} \mathrm{NO}_{2} \mathrm{SCr}\end{array}$ & $\begin{array}{c}63 \\
359 \\
452\end{array}$ & $\begin{array}{l}\text { endo } \\
\text { endo } \\
\text { endo }\end{array}$ \\
\hline $\mathrm{VOL}_{2}$ & $\begin{array}{c}33.7-164.8 \\
195-352 \\
384-699.4\end{array}$ & $\begin{array}{l}75.35 \\
263 \\
477\end{array}$ & $\begin{array}{l}16.592(16.823) \\
19.893(20.175) \\
50.024(52.903)\end{array}$ & $\begin{array}{c}86.509 \\
(89.901)\end{array}$ & $\begin{array}{c}-\mathrm{C}_{6} \mathrm{H}_{7} \mathrm{~N}_{2} \mathrm{O}_{2} \\
-\mathrm{C}_{6} \mathrm{H}_{7} \mathrm{~N}_{2} \mathrm{O}_{2} \\
-\mathrm{C}_{13} \mathrm{H}_{20} \mathrm{~N}_{4} \mathrm{O}_{3} \\
\mathrm{C}_{19}+\mathrm{V}\end{array}$ & $\begin{array}{c}66 \\
258 \\
489\end{array}$ & $\begin{array}{l}\text { End } \\
\text { End } \\
\text { end }\end{array}$ \\
\hline
\end{tabular}

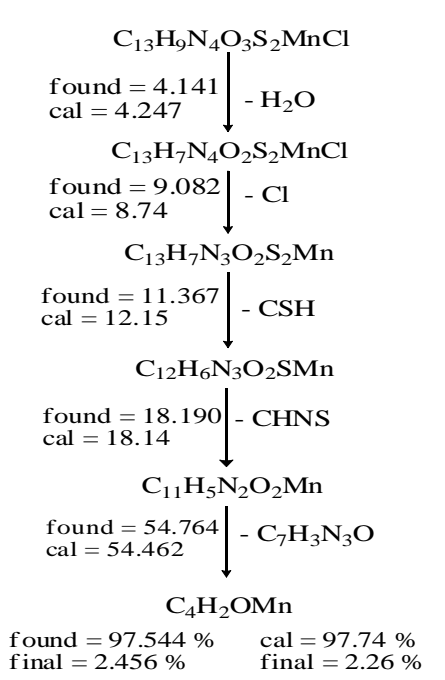

$\left[\mathrm{Mn} \mathrm{L1Cl}\left(\mathrm{H}_{2} \mathrm{O}\right)\right]$

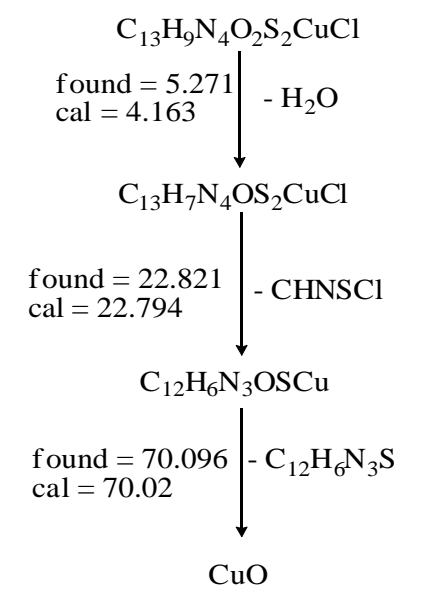

found $=98.188$ final $=1.812$

$$
\begin{aligned}
& \mathrm{C}_{13} \mathrm{H}_{10} \mathrm{~N}_{4} \mathrm{O}_{3} \mathrm{~S}_{2} \mathrm{CrCl}_{3} \\
& \begin{array}{l}
\text { found }=7.647 \\
\text { cal = 7.25 }
\end{array} \\
& \mathrm{C}_{13} \mathrm{H}_{8} \mathrm{~N}_{4} \mathrm{O}_{2} \mathrm{~S}_{2} \mathrm{CrCl}_{2.5} \\
& \begin{array}{l}
\text { found = }=31.601 \\
\text { cal = 32.303 }
\end{array} \\
& \mathrm{C}_{12} \mathrm{H}_{7} \mathrm{~N}_{3} \mathrm{O}_{2} \mathrm{SCr}+1 /{ }_{2} \mathrm{Cl} \\
& \mathrm{CHNSCl}_{2.5} \\
& \text { found = 52.31 } \\
& \text { cal = 53.009 }
\end{aligned}
$$
$\left[\mathrm{Cu} \mathrm{L1Cl}\left(\mathrm{H}_{2} \mathrm{O}\right)\right]$$$
\text { [Cr } \left.\mathrm{HL}_{1 \mathrm{Cl}}\left(\mathrm{H}_{2} \mathrm{O}\right)\right]
$$

cal $=92.562$ final $=7.48$

$$
\begin{gathered}
\text { found }=16.823 \mid-\mathrm{C}_{6} \mathrm{H}_{7} \mathrm{~N}_{2} \mathrm{O}_{2} \\
\begin{array}{c}
\mathrm{C}_{44} \mathrm{H}_{34} \mathrm{~N}_{8} \mathrm{O}_{7} \mathrm{~V} \\
\text { cal }=16.592
\end{array}
\end{gathered}
$$$$
\text { cal }=96.977 \text { found }=91.558
$$ 
Table (5) Thermodynamic Data of the Thermal Decomposition of Ligands and their Complexes.

\begin{tabular}{|c|c|c|c|c|c|c|c|c|c|c|}
\hline Sam (step) & T range ${ }^{\circ} \mathrm{C}$ & $\mathrm{n}$ & $\mathrm{R}^{2}$ & $\mathrm{~T}_{\max }{ }^{\circ} \mathrm{K}$ & $\begin{array}{c}\mathrm{E} \\
\mathrm{KJ} \mathrm{mol}^{-1}\end{array}$ & $\begin{array}{c}\Delta \mathrm{H}^{2} \\
\mathrm{KJ} \mathrm{mol}^{-1}\end{array}$ & $\begin{array}{c}\mathrm{Z} \\
\mathrm{s}^{-1} \times 10^{5}\end{array}$ & $\begin{array}{c}\Delta \mathrm{S}^{*} \\
\mathrm{~J} \mathrm{~mol}^{-1} \mathrm{~K}^{-1}\end{array}$ & $\begin{array}{c}\Delta \mathrm{G}^{*} \\
\mathrm{KJ} \mathrm{mol}^{-1}\end{array}$ & $\mathrm{~K} \times 10^{-7}$ \\
\hline $\mathrm{HL}_{1}(1)$ & $164.2-235.5$ & 1 & 0.99 & 210.1 & 7.666994 & 2.982 & 425.5 & -104.171 & 61.983 & 17.958 \\
\hline $\mathrm{HL}_{1}(2)$ & $235.5-599.5$ & 1 & 0.99 & 566.5 & 12.24048 & 5.6986 & 830.9 & $\begin{array}{l}-101.378 \\
\end{array}$ & 85.467 & 21.189 \\
\hline $\mathrm{HL}_{2}(1)$ & $199.1-313.7$ & 1 & 0.99 & 287.9 & 7.442325 & 2.56101 & 505.41 & -103.0818 & 68.4756 & 8.0847 \\
\hline $\mathrm{HL}_{2}(2)$ & $313.7-589.3$ & 1 & 0.99 & 524.4 & 11.90189 & 5.49496 & 794.69 & -101.5799 & 83.77449 & 20.9573 \\
\hline $\mathrm{CuL}_{1=1} 1$ & $33.5-132.8$ & 0.9 & 0.97 & 70.32 & 4.57425 & 1.736 & 124.3 & -117.54 & 41.86 & 3.9316 \\
\hline $\mathrm{CuL}_{1=2}$ & $133-433.7$ & 0.9 & 0.99 & 319 & 9.60396 & 4.509 & 479.0 & -103.878 & 68.160 & 15.4668 \\
\hline $\mathrm{CuL}_{1=} 3$ & $462.9-699.4$ & 0.9 & 0.99 & 611.6 & 12.68103 & 7.174 & 410.0 & -107.65 & 96.022 & 8.37053 \\
\hline $\mathrm{CrL}_{\mathrm{l}=1} 1$ & $33.6-147.6$ & 0.9 & 0.99 & 72.03 & 5.792853 & 2.9547 & 146.04 & -108.8958 & 40.12847 & 7.2377 \\
\hline $\mathrm{CrL}_{1=2}$ & $305.4-399.7$ & 0.9 & 0.99 & 359 & 12.11535 & 6.85467 & 477.78 & -104.1716 & 72.76927 & 9.8303 \\
\hline $\mathrm{CrL}_{1=3} 3$ & $430.1-699.3$ & 0.9 & 0.99 & 476 & 12.36905 & 5050709 & 977.70 & -100.4266 & 8.8394191 & 25.44185 \\
\hline $\mathrm{VOL}_{1}=1$ & $33.7-164.8$ & 0.9 & 0.99 & 75.35 & 6.609926 & 3.7215 & $\begin{array}{l}1087.817 \\
\end{array}$ & -92.370 & 35.9035 & 41.5445 \\
\hline $\mathrm{VOL}_{1}=2$ & $195-352$ & 0.9 & 0.99 & 263 & 9.66478 & 5.19925 & 359.8129 & $\begin{array}{l}-105.167 \\
\end{array}$ & 61.6855 & 10.011818 \\
\hline $\mathrm{VOL}_{1}=3$ & $384-699.4$ & 0.9 & 0.99 & 477 & 16.21645 & 9.97655 & 646.665 & $\begin{array}{l}-103.074 \\
\end{array}$ & 87.336 & 8.3455 \\
\hline $\mathrm{MnL}_{1}=1$ & $51.6-109.3$ & 0.9 & 0.99 & 95.13 & 6.289215 & 2.84248 & 203.67145 & -107.7458 & 47.510686 & 10.3174 \\
\hline $\mathrm{MnL}_{1}=2$ & $178.8-257.9$ & 0.9 & 0.99 & 241.5 & 7.706092 & 3.4988 & $\begin{array}{l}314.8201 \\
\end{array}$ & -105.7825 & 57.03 & 12.9757 \\
\hline $\mathrm{MnL}_{1}=3$ & $297-408.9$ & 0.9 & 0.99 & 358.8 & 10.67851 & 5.239813 & 534.23834 & -103.5197 & 72.95827 & 14.9305 \\
\hline $\mathrm{MnL}_{1}=4$ & $408.9-699.6$ & 0.9 & 0.99 & 467.8 & 13.42782 & 6.36765 & 926.9402 & -101.1067 & 722.6238 & 21.4988 \\
\hline
\end{tabular}

$\mathrm{R}^{2}=$ correlation coefficient of the linear plot, $n=$ order of reaction, $\mathrm{Z}=$ preexponential factor

\section{Theoretical Study}

The vibration spectra Azo dyes ligands are calculated by using a Hyber chem. 8 method. The results obtained for wave numbers are presented in Table (6), and the comparison with the experimental values indicates some deviations. These deviations may be due to the harmonic oscillator approximation and lack of electron correlation. It is reported [25] that frequencies coupled with (HFT) approximation and quantum harmonic oscillator approximations tend to be $10 \%$ too high. And structures of ligands are calculated to search for the most probable model building stable structure. These shapes show the calculated optima geometries for compounds prepared as shown in Figure (4). And the $\Delta \mathrm{E}_{\mathrm{b}}$ and $\Delta \mathrm{H}_{\mathrm{f}}$ for compounds in table (7).

Table (6) Comparison of Experimental and Theoretical Vibration Frequencies for Ligands

\begin{tabular}{|c|c|c|c|c|c|c|c|c|c|c|}
\hline Ligand & $v(\mathrm{OH})$ & $\begin{array}{c}v(\mathrm{CH}) \\
\text { aromatic }\end{array}$ & $\begin{array}{l}v(\mathrm{CH}) \\
\text { aleph }\end{array}$ & $\begin{array}{c}v(\mathrm{C}-\mathrm{H}) \\
\text { aldehyde }\end{array}$ & $v(C-S)$ & $v(\mathrm{C}=\mathrm{N})$ & $v(\mathrm{~S}-\mathrm{H})$ & $\begin{array}{c}v(\mathrm{C}=\mathrm{O}) \\
\text { aldehyde }\end{array}$ & $\begin{array}{c}v(\mathrm{C}=\mathrm{O}) \\
\text { ring }\end{array}$ & $v(\mathrm{~N}=\mathrm{N})$ \\
\hline $\mathrm{HL}_{1}$ & $\begin{array}{c}* 3566 \\
* * 3600 \\
* * *-0.95\end{array}$ & $\begin{array}{c}* 3076 \\
* * 3110 \\
* * *-1.10\end{array}$ & - & $\begin{array}{l}* 2889 \\
* * 2800 \\
* * * 3.17\end{array}$ & $\begin{array}{cr}* 660 & * 653 \\
* * 680 & * * 621 \\
& * * *-3.03 \\
& * * * 4.90\end{array}$ & $\begin{array}{c}* 1635 * 1575 \\
* * 1643 * * 1455 \\
* * *-0.4 \\
* * * 7.61\end{array}$ & $\begin{array}{c}* 2597 \\
* * 2553 \\
* * * 1.69\end{array}$ & $\begin{array}{c}* 1645 \\
* * 1656 \\
* * *-0.66\end{array}$ & - & $\begin{array}{c}* 1520 * 1430 \\
* * 1400 * * 1390 \\
* * * 7.89 \\
* * * 2.79\end{array}$ \\
\hline $\mathrm{HL}_{2}$ & $\begin{array}{c}* 3429 \\
* * 3488 \\
* * *-1.720\end{array}$ & $\begin{array}{c}* 3055 \\
* * 3090 \\
* * *-1.146\end{array}$ & $\begin{array}{c}* 2893 \\
* * 2888 \\
* * * 0.173\end{array}$ & $\begin{array}{c}* 2924 \\
* * 2943 \\
* * *-0.650\end{array}$ & - & - & - & $\begin{array}{c}* 1636 \\
* * 1647 \\
* * *-0.672\end{array}$ & $\begin{array}{c}* 1643 \\
* * 1650 \\
* * *-0.426\end{array}$ & $\begin{array}{c}* 1481 * 1419 \\
* * 1470 \\
* * *-1.379\end{array}$ \\
\hline
\end{tabular}

*Experimental frequency. $* *$ Theoretical frequency. $* * *$ Error\% due to main difference in the experimental measurements and theoretical treatments of vibration spectrum.

Table (7) Conformation Energies in $\left(\mathrm{kJ} \mathrm{mol}^{-1}\right)$ for the Ligands and its Metal Complexes

\begin{tabular}{|c|c|c|c|c|c|}
\hline \multirow{2}{*}{ Compounds } & \multicolumn{2}{|c|}{$\mathrm{PM} 3$} & \multirow{2}{*}{ Compounds } & \multicolumn{2}{|c|}{$\mathrm{PM} 3$} \\
\cline { 2 - 3 } & $\Delta \mathrm{H}_{f}{ }^{\circ}$ & $\Delta \mathrm{E}_{b}$ & & $\Delta \mathrm{H}_{f}{ }^{\circ}$ & $\Delta \mathrm{E}_{b}$ \\
\hline $\mathrm{HL}_{1}$ & 117.873 & -3224.430 & $\mathrm{HL}_{2}$ & 36.82307 & -5291.2765 \\
\hline $\mathrm{CrL}_{1}$ & 15.3232 & -4321.657 & $\mathrm{CrL}_{2}$ & 54.873 & -6443.3034 \\
\hline $\mathrm{MnL}_{1}$ & 88.49704 & -997.4150296 & $\mathrm{MnL}_{2}$ & 210.8897177 & -5927.125282 \\
\hline $\mathrm{CoL}_{1}$ & 21.4043223 & -976.5386777 & $\mathrm{CoL}_{2}$ & 750.3750690 & -7634.2229310 \\
\hline $\mathrm{CuL}_{1}$ & 65.543243 & -4675.322179 & $\mathrm{CuL}_{2}$ & 132.76597 & -7652.546235 \\
\hline $\mathrm{NiL}_{1}$ & 23.314242 & -6543.646497 & $\mathrm{NiL}_{2}$ & 33.76855 & -4567.834 \\
\hline $\mathrm{VOL}_{1}$ & 43.658 & -5256.7683 & $\mathrm{VOL}_{2}$ & 87.9665 & -3445.678 \\
\hline
\end{tabular}




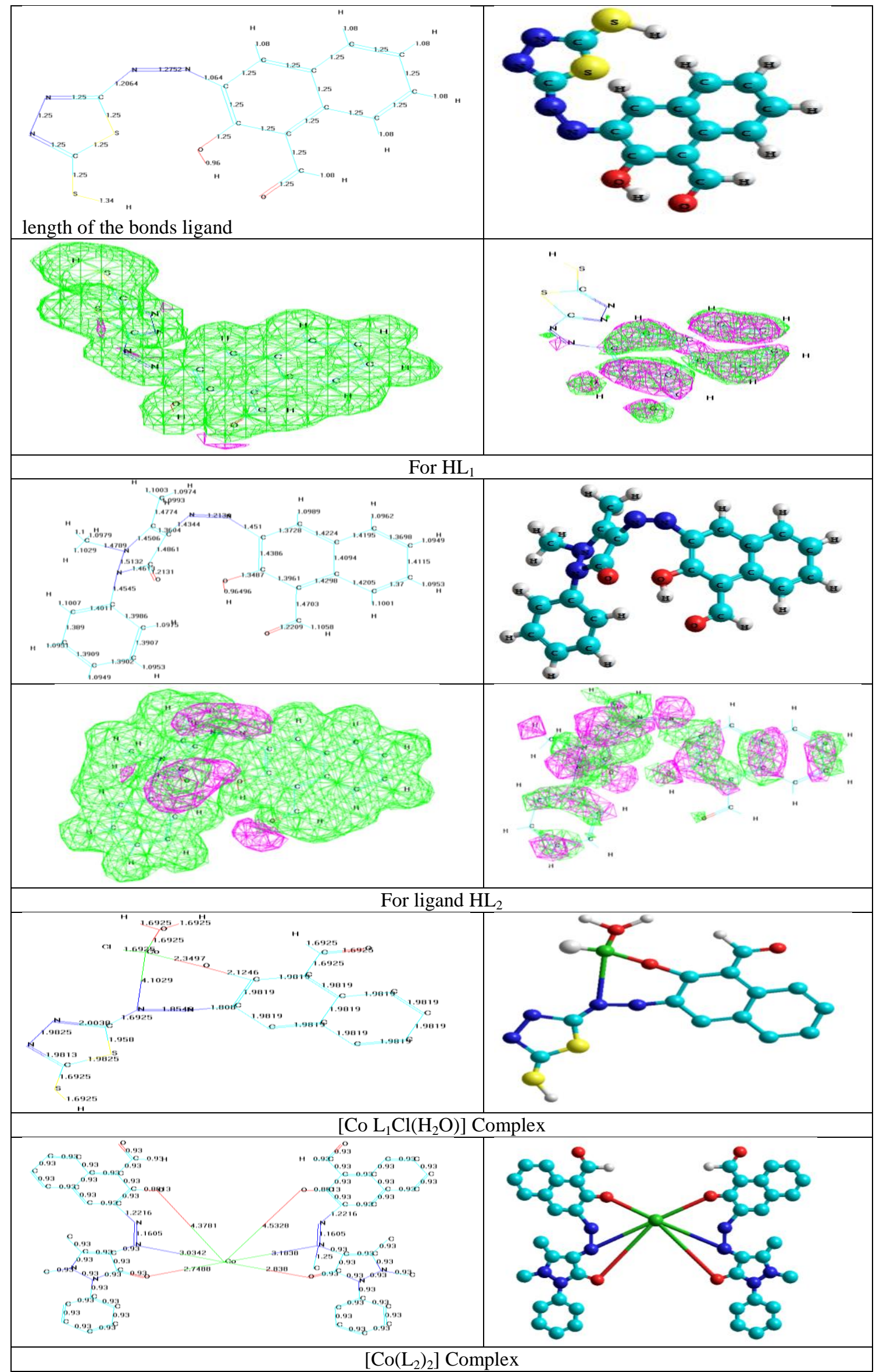

Fig. (4) Conformational Structures of Ligands and Cobalt Complexes. 


\section{Microbiological Investigation}

The biological activity of ligands and their metal complexes is tested against bacteria; we use more than one test organism to increase the chance of detecting antibiotic principles in tested materials. The organisms used in the present investigation includes two Gram positive bacteria (B. subtilis and $S$. aureus) and two Gram negative bacteria
(E. coli and P. aeruginosa). The results of the bactericidal screening of the synthesized compounds are recorded in Table (8). An influence of the central ion of the complexes in the antibacterial activity against the tested Gram positive and Gram negative organisms shows that the complexes have an enhanced activity compared to the ligand itself.

Table (8) Antibacterial Activity Data of Ligands and their Complexes as Inhibition Zone (mm).

\begin{tabular}{|c|c|c|c|c|}
\hline Compound & Bacillus subtilis $G+$ & Staphylococcus aureus ${ }^{+}+$ & Escherichia coli $G^{-}$ & Pseudomonas aeruginosa $G^{-1}$ \\
\hline $\mathrm{HL}_{1}$ & 18 & 16 & 19 & 18 \\
\hline $\mathrm{HL}_{2}$ & 25 & 19 & 19 & 24 \\
\hline $\mathrm{VOL}_{1}$ & 18 & 23 & 22 & 20 \\
\hline $\mathrm{VOL}_{2}$ & 22 & 29 & 22 & 18 \\
\hline $\mathrm{CrL}_{1}$ & 12 & 10 & 9 & 7 \\
\hline $\mathrm{CrL}_{2}$ & 12 & 13 & 21 & 16 \\
\hline $\mathrm{MnL}_{1}$ & 22 & 21 & 23 & 28 \\
\hline $\mathrm{MnL}_{2}$ & 26 & 15 & 12 & 21 \\
\hline $\mathrm{CoL}_{1}$ & 20 & 21 & 24 & 22 \\
\hline $\mathrm{CoL}_{2}$ & 26 & 9 & 23 & 22 \\
\hline $\mathrm{NiL}_{1}$ & 15 & 23 & 21 & 26 \\
\hline $\mathrm{NiL}_{2}$ & 17 & 22 & 24 & 26 \\
\hline $\mathrm{CuL}_{1}$ & 20 & 22 & 19 & 24 \\
\hline $\mathrm{CuL}_{2}$ & 21 & 22 & 23 & 2 \\
\hline
\end{tabular}

Key to interpretation: Less than $10 \mathrm{~mm}=$ inactive, $10-15 \mathrm{~mm}=$ weakly active, $15-20$ $\mathrm{mm}=$ moderately active, more than $20 \mathrm{~mm}=$ highly active.

\section{References:}

[1]Savic, J. and Vasic, S. 2006. Complex Formation Between Pd(II) and Immobilized Imidazol Azo Chromotropic Acid. Acta Chim. Slov.; 53(12): 36-42.

[2]Maradiya, H. R. 2001. Synthesis of Azobenzo (b) Thiophene Derivatives and Their Dyeing Performance on Polyester Fiber. Turk.J.Chem; 25 : 441-450.

[3]Jarad, A.J. 2012. Synthesis and Characterization of New Azo Dye Complexes with Selected Metal Ions. J. of Al-Nahrain Uni.; 15(4):74-81.

[4]Karipcin, F. and Kabalcilar, E. 2007. Spectroscopic and Thermal Studies on Solid Complexes of 4-(2Pyridylazo)-resorcinol with Some Transition Metals. Acta. Chim.Slov; 54(6):242-247.

[5]Patel, V. H.; Petal, M. P. and Patel, R. G. 2002. Synthesis and
Application of Novel Hetrocyclic Dyes Based on 11-amino-13-Hacenaphtho[1,2-e] Pyridazin[3,2b]Quinazolin-13-one. J. Serb. Chem. Soc; 67 (11): 727-737.

[6]Kupradinun, P.; Rienkijakaru, M.; Tanyakaset, M.; Tepsuwan, A. and Kusamran, W. R. 2002. Carcinogenicity Testing of the Cosmetic Dye:D and C Red No.36. Asi. Pac. J. of Canc. Pre.; 3: 55-60.

[7]Maradiya, H. R. and Patel, V. S. 2001. Synthesis and Dyeing Performance of Some Novel Hetrocyclic Azo Disperse Dyes. J. Braz. Chem. Soc; 12 (6) : 1-6.

[8]Gregory, P.; Waring, D. R. and Hallos, G. 1990. The Chemistry and Application of Dyes. plenum press, London. : 18-20.

[9]Kutgen, H. K. and Heren, Z. 1998. Thermal Behaviour of Some Azo Dyes Containing Sterically Hindered 
and Water Soluble Groups. Turk. J. Chem.; 22 : 403-408.

[10] Pal, S. and Sinho, C. 2001. Studies on the Reactivity of cis$\mathrm{RuCl} 2$ Fragment in $\mathrm{Ru}(\mathrm{pph} 3)$ 2(TaiMe) $\mathrm{Cl} 2$ with $\mathrm{N}, \mathrm{N}-\mathrm{Chelators}$ (Taime $=1-$ Methyl-2-(p-Tolylazo)

Imidazole). Spectral and Electrochemical Characterization of the Products, Proc. Ind. Acad. Sci.; 113(3):173-182.

[11] Carballo, R.; Castineiras, A.; Covelo, B.; Niclos, J. and VazguezLopez, E. 2001. Synthesis and Characterization of Potassium Complex of Magneson: [K(HL) (OH2)2] [H2L=4-(4-nitrophenylazo) resorcinol (Magneson)], Polyhedron.; 20(3): 2415-2420.

[12] Shujuan, S.; Hongxiang, L.; Gao, Y.; Fan, P.; Ma, B.; Ge, W. and Wang, X. 2004. Liquid chromatography-tandem mass spectrometric method for the analysis of fluconazole and evaluation of the impact of phenolic compounds on the concentration of fluconazole in Candida albicans. J. Pharm. Biomed. Anal.; 34 : 1117-1124.

[13] Dixit, B.; Patel, H.; Dixit, R. and Desai, D. 2010. Synthesis, characterization and dyeing assessment of novel acid azo dyes and mordent acid azo dyes on 2hydroxy-4-methoxybenzophenone on wool and silk fabrics. J. Serb. Chem. Soc.; 75 (5): 605-614.

[14] Al-Hamdani, A. A. S. and Shaker Sh. A. 2011. Synthesis, characterization, Stuctural Studies and Biological Activity of a New Schiff Base-Azo Ligand and its Complexation with Selected Metal Ions. Oriental J. Chem.; 27 (3): 825845.

[15] Nakamoto, N. 2009. Infrared and Raman Spectra of Inorganic and Coordination Compounds, $6^{\text {th }} \mathrm{Ed}$, Part 2 John wiley and Sons, Inc., New Jersy.
[16] Shaker, Sh. A.; Yang, F. and AlHamdani, A. A. S. 2009. Synthesis and Characterization of Mixed Ligand Complexes of 8Hydroxyquinoline and ohydroxybenzylidene-1-phenyl-2,3dimethyl-4-amino-3-pyrazolin-5-on with $\mathrm{Fe}(\mathrm{II}), \mathrm{Co}(\mathrm{II}), \mathrm{Ni}(\mathrm{II})$ and $\mathrm{Cu}(\mathrm{II})$ ions. European J. Scie. Resea.; 33(4): 702-709.

[17] Sliverstein, R. M. and Webser, X. F. 2005. Spectrometric Identification of Organic Compounds. $7^{\text {th }}$ Ed., Jon Wiley and Son, Inc. USA.

[18] Lever, A. B. P. 1968. Inorganic Electronic Spectroscopy. New York. 6. 121.

[19] Al-Hamdani, A. A. S. 2013. Metal Complexes of Multidentate Schiff Base-Azo Ligand: Synthesis, Characterization and Biological Activity. Dirasat, Pure Scie.; 39 (1): 61-72.

[20] Shaker, Sh. A. 2010. Preparation and Spectral Properties of MixedLigand Complexes of VO(II), Ni(II), $\mathrm{Zn}(\mathrm{II}), \mathrm{Pd}(\mathrm{II}), \mathrm{Cd}(\mathrm{II})$ and $\mathrm{Pb}(\mathrm{II})$ with Dimethylglyoxime and NAcetylglycine. E-J. Chem.; 7 (4): 1598-1604.

[21] Al-Hamdani, A. A. S. and AlZoubi, W. 2015. New metal complexes N3 tridentate ligand: Synthesis, spectral studies and biological activity. Spectrochimica Acta Part A: Mole. and Biomol. Spect., $137:$ 75-89.

[22] Al-Hamdani, A. A. S.; Jarad, A. J. and Al-Atrakchi, S. A. 2009. Synthesis and spectral analysis of $\mathrm{VO}(\mathrm{II}), \mathrm{Cr}(\mathrm{II}), \mathrm{Zn}$ (II), $\mathrm{Cd}(\mathrm{II}), \mathrm{Hg}$ (II) and $\mathrm{UO}_{2}$ complexes with mixed ligands of bipyridyl and novel Schiff base. Diala. J. 36: 349-361.

[23] Coats, A. W. and Redfern, J. P.1964. Kinetic parameters from thermogravimetric data. Nature, 201: 68-79. 
[24] Frost, A. A. and Pearson, R. G.1964. Kinetics and Mechanism $2^{\text {nd }}$ Ed., with permission of john wiley \& sons, Inc Wiley, New York.
[25] Baker, Sh. R. 2012. Synthesis, Spectral study and biological activity of some metal ions complexes with bidentate ligands. J. AL-Nahra. Uni. Sci.; 15 (3): 30-42.

\section{دراسات طيفية وتحليل حراري لليكاندات أصباغ آزو جديدة و معقداتها مع بعض الإن ايونات الفلزات الانتقالية}

زينب عبد الهادي حسن

\author{
عباس علي صالح الحمداني \\ قسم الكيمياء، كلية العلوم للبنات، جامعة بغداد، العراق
}

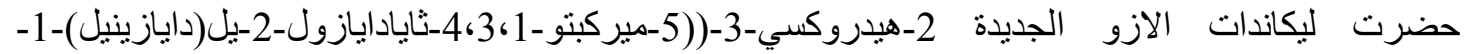

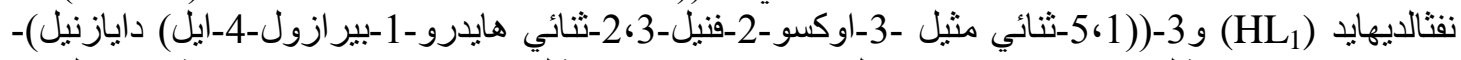

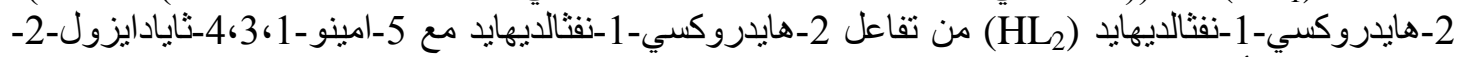

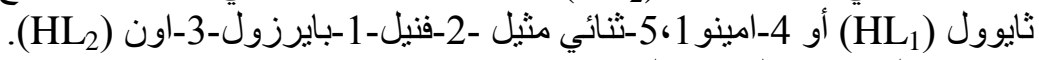
حضرت المعقدات بالصيغة العامة ]

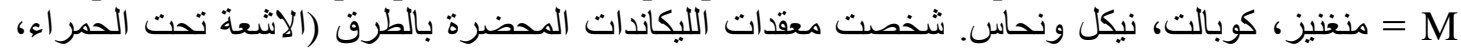

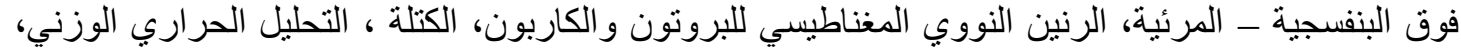

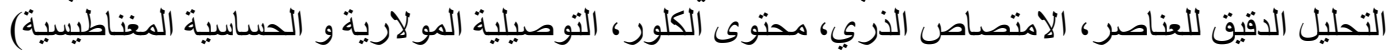

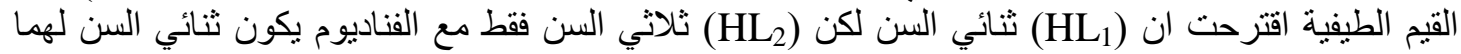

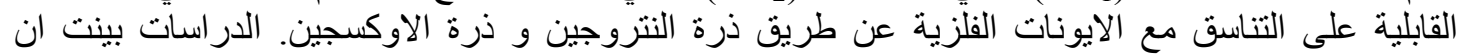

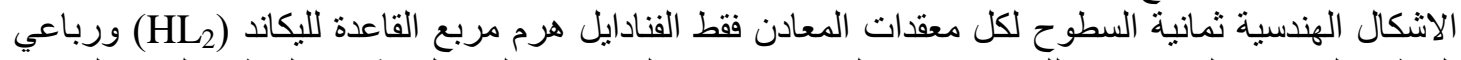

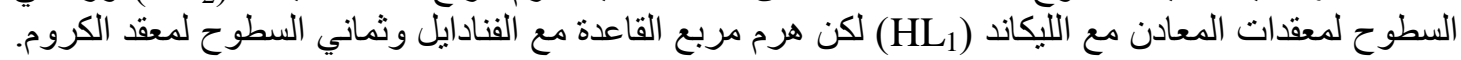

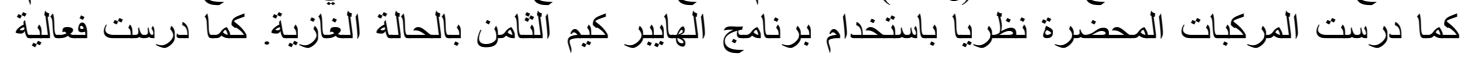
المركبات المحضرة ضد البكرئ البكتريا.

الكلمات المفتاحية: ليكاندات ثنائية وثلاثية، ليكاندات أزو، تحلل حر اري و الثوابت الحركية والثرموديناميكية 